\title{
Combining simultaneous density and velocity measurements of rotor blade tip vortices under cyclic pitch conditions
}

\author{
Johannes N. Braukmann ${ }^{1}$ (D) . Andreas Goerttler ${ }^{1}$ (D) $\cdot$ C. Christian Wolf ${ }^{1}$ D $\cdot$ Clemens Schwarz $^{1}$ (D) Markus Raffel $^{1}$ (D)
}

Received: 4 May 2021 / Revised: 9 July 2021 / Accepted: 2 August 2021 / Published online: 6 September 2021

(C) The Author(s) 2021

\begin{abstract}
An investigation into blade tip vortices of a sub-scale rotor under cyclic pitch conditions is carried out. Background oriented schlieren (BOS), particle image velocimetry (PIV), and computational fluid dynamics (CFD) are applied to the same test cases. This approach allows to combine the velocity data from PIV in a measurement plane, the density related data from BOS in a measurement volume, and the comprehensive set of flow variables provided by unsteady detached eddy simulations. Vortices up to an age of $\Psi_{\mathrm{v}}=70^{\circ}$ in case of PIV and CFD, and up to $\Psi_{\mathrm{v}}=200^{\circ}$ in case of BOS are considered. The vortex locations are obtained through all three techniques. The unsteadiness of the vortices was obtained by the experimental results, whereas CFD provides an average solution. An increased position scatter was observed during the downstroke of the pitch cycle with both experimental methods and was found to be in good agreement. In the second part, the PIV velocity data are compared to common vortex models. An approach to link the density distribution and the swirl velocity is applied to the measured data. Based on the CFD results, it is shown that the assumption of isothermal flow yields better agreement between velocity and density than isentropic flow.
\end{abstract}

\section{Graphic abstract}

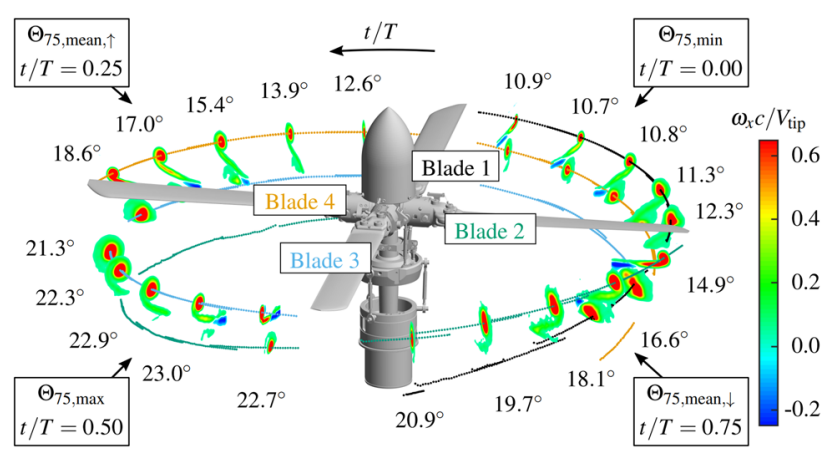

Keywords Blade tip vortices $\cdot$ BOS $\cdot$ PIV $\cdot$ Experiments

\section{List of symbols}

a Major ellipse axis (m)

$b \quad$ Minor ellipse axis (m)

$c \quad$ Chord $(c=0.072 \mathrm{~m})$

$C_{\mathrm{T}} \quad$ Rotor thrust coefficient $\left(C_{\mathrm{T}}=F_{z} /\left(\rho A \Omega^{2} R^{2}\right)\right)$

$f \quad$ Focal length $(\mathrm{m})$

Johannes N. Braukmann

johannes.braukmann@dlr.de

1 German Aerospace Center (DLR), Institute of Aerodynamics and Flow Technology, Bunsenstr. 10, Göttingen, Germany $f_{\#} \quad$ F-number

$F_{z} \quad$ Thrust (N)

I Intensity (counts)

$k \quad$ Reduced frequency $k=\Omega c /\left(2 V_{\text {tip }} r / R\right)$

$=\Omega c /(2 \Omega r)=c /(2 r)$

$L \quad$ Length (m)

Ma Mach number

Integer factor

$\begin{array}{ll}n_{\mathrm{p}} & \text { Polytropic exponent } \\ N_{\mathrm{b}} & \text { Number of blades }\left(N_{\mathrm{b}}\right.\end{array}$

$N_{\mathrm{b}} \quad$ Number of blades $\left(N_{\mathrm{b}}=4\right)$

$p \quad$ Pressure $(\mathrm{Pa})$ 
$p_{\infty} \quad$ Ambient pressure $(\mathrm{Pa})$

$r \quad$ Radial coordinate in the vortex coordinate system $(\mathrm{m})$

$r_{\mathrm{c}} \quad$ Vortex core radius $(\mathrm{m})$

$r_{\text {max shift }}$ Radius of maximum BOS shift (m)

$r_{\mathrm{r}} \quad$ Radial coordinate in the rotor coordinate system (m)

$r_{\text {void }} \quad$ Radial extent of the particle void $(\mathrm{m})$

$R \quad$ Rotor radius $(R=0.65 \mathrm{~m})$

Re Chord based Reynolds number

$s \quad$ Displacement (px)

$s_{\mathrm{c}} \quad$ Cell size of the CFD grid (m)

$S \quad$ BOS sensitivity factor (m)

$t \quad$ Time (s)

$T \quad$ Period of the pitch oscillation (s)

$T_{\mathrm{v}} \quad$ Temperature in the vortex $(\mathrm{K})$

$T_{\infty} \quad$ Ambient temperature (K)

$u, v, w \quad$ Velocity components in $x, y, z$-direction $(\mathrm{m} / \mathrm{s})$

$V_{\text {tip }} \quad$ Blade tip velocity $\left(V_{\text {tip }}=96.4 \mathrm{~m} / \mathrm{s}\right)$

$V_{\theta} \quad$ Swirl velocity $(\mathrm{m} / \mathrm{s})$

$V_{\infty} \quad$ Axial inflow velocity $\left(V_{\infty}=2.6 \mathrm{~m} / \mathrm{s}\right)$

$x, y, z \quad$ Cartesian coordinates (m)

$z_{\mathrm{r}} \quad$ Axial coordinate in the rotor coordinate system (m)

$z \quad$ Axial coordinate in the vortex coordinate system (m)

$\beta \quad$ Turbulence factor

$\gamma \quad$ Isentropic exponent $(\gamma=1.4)$

$\Gamma_{\infty} \quad$ Total vortex circulation $\left(\mathrm{m}^{2} / \mathrm{s}\right)$

$\Gamma_{0.5 c} \quad$ Vortex circulation at $r / c=0.5\left(\mathrm{~m}^{2} / \mathrm{s}\right)$

$\Theta \quad$ Blade pitch angle $\left({ }^{\circ}\right)$

$\Lambda \quad$ Aspect ratio $(\Lambda=R / c \approx 9)$

$\rho \quad$ Density $\left(\mathrm{kg} / \mathrm{m}^{3}\right)$

$\rho_{\infty} \quad$ Ambient density $\left(\mathrm{kg} / \mathrm{m}^{3}\right)$

$\sigma \quad$ Rotor solidity $\left(\sigma=N_{\mathrm{b}} c /(\pi R)=0.1025\right)$

$\Psi_{\mathrm{v}} \quad$ Vortex age $\left({ }^{\circ}\right)$

$\Omega \quad$ Angular velocity of the rotor ( $\mathrm{rad} / \mathrm{s})$

\section{Introduction}

Blade tip vortices are a dominant feature of rotor wakes. They form a complex, helical structure and are the cause for many undesired effects: Their interaction with blades or other vortices can lead to increased acoustic emissions, trigger stall on the blades, or may give rise to vibrations in the rotorcraft.

Older measurements used visualization techniques (Gray 1956) or point wise methods as, for example, laser doppler velocimetry measurements (Scully and Sullivan 1972). A major advancement was achieved by the use of particle image velocimetry (PIV), which is a technique to measure velocities in a plane or even in a volume. PIV made it possible to access the entire velocity field of a vortex at one instantaneous measurement. Since then, many investigations of blade tip vortices based on PIV have been carried out, ranging from model to full-scale applications (Heineck et al. 2000; McAlister 2004; Schwarz et al. 2020; De Gregorio et al. 2021; Uluocak et al. 2021). An overview of the contribution of PIV to helicopter aerodynamics is given by Raffel et al. (2017). A drawback of the technique is the dependency on the tracking-accuracy of the tracer particles, a lack of particles in vortex flows, and the high effort when applying PIV to full-scale measurements outside the laboratory.

The background oriented schlieren technique (BOS) offers an alternative, density-based measurement technique for vortices. Comparable to classic schlieren techniques, BOS obtains a result that is linked to the density gradient of a transparent, compressible medium (Dalziel et al. 2000). The main advantage compared to classic schlieren setups is the reduced complexity and the feasibility of measurements outside of a laboratory environment. The initial publication by Raffel et al. (2000b) in 2000 demonstrated the applicability of BOS to blade tip vortices in a full-scale experiment on a helicopter. Since then, the technique was used in many different tip vortex investigations (Heineck et al. 2010; Schairer et al. 2013; Schwarz et al. 2020). With BOS, it is possible to detect the positions of blade tip vortices in the rotor wake and even reconstruct the three-dimensional (3D) positions based on a setup with multiple cameras (Bauknecht et al. 2014). In experiments focusing on the vortex positions, a detailed assessment of the vortex topology is often not included. Other experiments mainly concentrate on the reconstruction of the actual density field using advanced reconstruction approaches (Atcheson et al. 2008; Berger et al. 2009; Nicolas et al. 2015).

The main advantages of BOS are the easy applicability even to full-scale and in-flight measurements, and the ease of obtaining data in a measurement volume. Hence, a combination of BOS and PIV not only results in data on both velocity and density, but also ensures to benefit from the advantages of both techniques. Combined PIV and BOS measurements were taken, for example, by Tokgoz et al. (2012), who simultaneously measured the temperature and the velocity in a thin fluid layer. More recently, Schwarz et al. (2020) applied PIV and BOS to the wake of a helicopter in ground effect, in both model- and full-scale.

The current study is based on simultaneous BOS and PIV measurements of blade tip vortices of a sub-scale rotor in the rotor test facility Göttingen (RTG) under cyclic pitch conditions. An earlier investigation based on PIV data of a different cyclic test case (Braukmann et al. 2020) showed that it is possible to reconstruct the azimuth-dependent downwash of the RTG rotor by means of PIV and also demonstrated the usability of the BOS data to add valuable information to the velocity fields. In addition, the data 


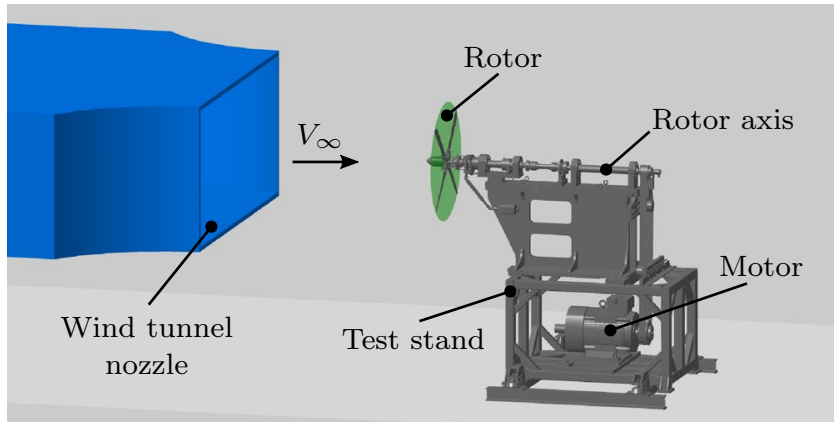

Fig. 1 Isometric model of the rotor test stand and the wind tunnel nozzle

from computational fluid dynamics (CFD) calculations of the same test case are available, allowing for a comprehensive analysis of the rotor wake and the blade tip vortices (Goerttler et al. 2020). The comparability of CFD and experiment is established, and thereafter the CFD results are used to link both experimental techniques, providing insight that could not have been obtained by the experimental data alone.

\section{Experimental setup}

\subsection{Rotor test stand Göttingen (RTG)}

The measurements were taken at the rotor test stand Göttingen (RTG). The facility features a rotor with a radius of $R=0.65 \mathrm{~m}$ and a horizontal axis (see Fig. 1). The purpose of the facility is to enable aerodynamic testing with optical measurement techniques at known conditions and a minimum blockage of the rotor downwash. A wind tunnel provides a defined axial inflow of $V_{\infty}=2.6 \mathrm{~m} / \mathrm{s}$, which leads to well-known and reproducible conditions during the measurements and convects the turbulent wake downstream, avoiding recirculation.

The four blades have a DSA-9A airfoil with a chord length of $c=72 \mathrm{~mm}$, a parabolic ONERA SPP8 blade tip planform and a negative linear twist of $-9.3^{\circ}$ along the aerodynamically relevant span between $0.25<r / R<1.00$. The profile and planform of the blades are shown in Fig. 2. The aspect ratio of the blades is $\Lambda=R / c \approx 9$, and the solidity $\sigma=N_{b} A_{b} /\left(\pi R^{2}\right)=0.1025$, with the number of blades $N_{b}=4$ and the area of a single blade $A_{b}=0.034 \mathrm{~m}^{2}$.

A balance, measuring all three forces and moments of the rotor, Hall-effect sensors measuring the blade pitch angle, and temperature and humidity sensors have been used to quantify the operating conditions of the rotor. A detailed description of the RTG can be found in (Schwermer et al. 2016).

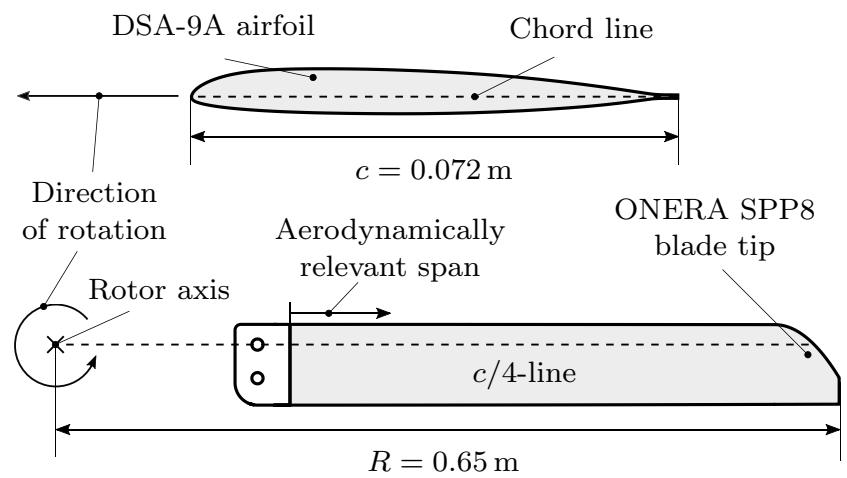

Fig. 2 Sketch of the DSA-9A profile and the blades' planform, both sketches at different scales

Table 1 Parameters of the measured test case

\begin{tabular}{lllll}
\hline$\Omega / 2 \pi$ & $V_{\text {tip }}$ & $\mathrm{M}_{75}$ & $\mathrm{Re}_{75}$ & $k_{75}$ \\
\hline $23.6 \mathrm{~Hz}$ & $96.4 \mathrm{~m} / \mathrm{s}$ & 0.21 & $3.6 \cdot 10^{5}$ & 0.074 \\
\hline
\end{tabular}

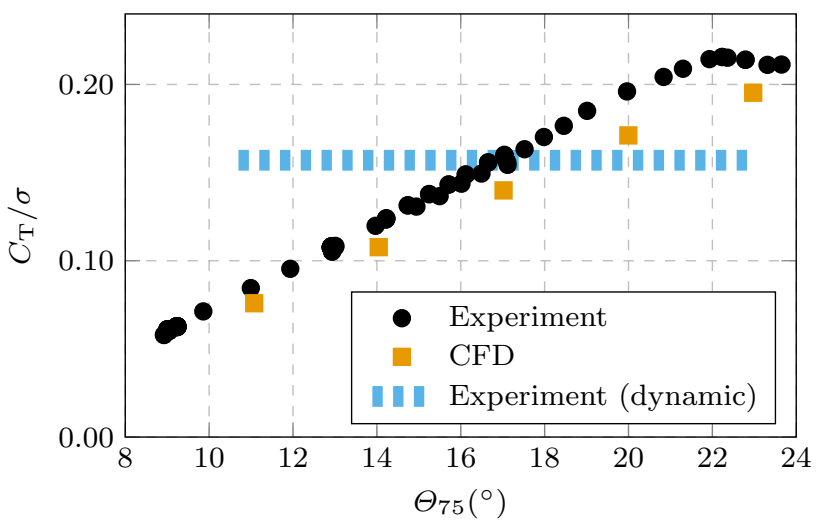

Fig. 3 Blade loading over pitch angle at an angular velocity of $\Omega=2 \pi \cdot 23.6 \mathrm{~Hz}$ and an inflow velocity of $V_{\infty}=2.6 \mathrm{~m} / \mathrm{s}$

\subsection{Test case}

The current investigation is based on measurements at a rotational frequency of $23.6 \mathrm{~Hz}$. The resulting Machand Reynolds numbers, and the reduced frequency at $r / R=0.75$ are given in Table 1 .

Figure 3 shows the static thrust polar (black dots) by means of the blade loading $C_{\mathrm{T}} / \sigma=F_{z} /\left(\rho_{\infty} \pi R^{2} \Omega^{2} R^{2} \sigma\right)$, with the thrust $F_{z}$, and the ambient density $\rho_{\infty}$. The pitch angle is given at a radius of $r / R=0.75$. The expected linear trend for attached flow conditions in Fig. 3 is met below $\Theta_{75}=22^{\circ}$. Above this value, static stall occurs. The maximum blade loading is 0.216 . The values obtained from CFD simulations by Goerttler et al. (2020) are added as orange squares. The simulation underpredicts 
the thrust coefficient compared to the experimental data, depending on the pitch angle.

The current investigation is based on a cyclic test case with a pitch cycle defined by

$\Theta_{75}=16.8^{\circ}-6.1^{\circ} \cos (2 \pi t / T)$, with $T=2 \pi / \Omega$.

The range of the cyclic pitch variation is indicated as a blue dashed line in Fig. 3. The chosen pitch setting provides a wide range of lift production: The flow over the blades is fully attached during the entire dynamic pitch cycle, even though the maximum pitch angle of $22.9^{\circ}$ exceeds the static stall angle. This is due to the fact that dynamic stall is delayed by unsteady aerodynamic effects (Mulleners and Raffel 2011). The mean thrust of this pitch setting is $F_{z}=236 \mathrm{~N}$ and the resulting mean blade loading $C_{\mathrm{T}} / \sigma=0.157$.

The cyclic pitch setting leads to an azimuthal dependency of the blade loading. Low pitch angles, which go along with a low blade loading, result in a low induced velocity and, thus, a reduced convection rate of weak blade tip vortices compared to high pitch angles. The resulting rotor wake is also azimuth-dependent as well as asymmetric. For the current investigation, three snapshots of the resulting rotor wake were taken with optical measurement systems. Since the field of view (FOV) of these systems was not large enough to capture the entire rotor wake at once, a scanning technique was applied: A key feature of the RTG is the ability to slowly rotate the usually stationary part of the swash plate. With this feature, it is possible to vary the phase of the pitch cycle at a certain position, e.g., relative to a fixed FOV of an optical measurement system. With this approach, it is possible to scan through the pitch cycle of the rotor without moving the optical measurement system. By additionally setting the captured vortex age according to the corresponding phase, snapshots of the rotor wake resulting from this cyclic pitch setting can be reconstructed. In the current investigation, the scan comprises recordings from 20 discrete phase positions. For each measurement, 200 images have been taken with each optical system. Based on this data, the mean shape and the aperiodic behavior of the rotor wake can be studied.

The axial inflow at the RTG represents slow climb conditions, whereas a cyclic pitch relates to maneuver or forward flight. A change in dynamic pressure, as it occurs in forward flight, is not reproduced. Therefore, the measurement conditions at the RTG represent generic test cases, which are suitable to study the influence of different pitch settings and flow conditions with a reduced measurement effort, and to compare the results from different measurement techniques as well as from corresponding numerical simulations.

\subsection{Optical measurement systems}

For the current investigation, both a stereoscopic background oriented schlieren (BOS) setup and a stereoscopic particle image velocimetry (PIV) setup have been used to simultaneously capture the tip vortices of the RTG. Based on these two techniques, it is possible to connect the densityrelated BOS shift to the velocities, that were measured with the PIV system, to gain an increased understanding of the vortices. In addition to these two optical systems, a camera focused on the blade tip is used. With this additional camera, it is possible to evaluate lead/lag and flap movements of the tip and the blade tip pitch angle. The obtained data of the vortex positions can, therefore, be derived with respect to the blade tip position.

An overview of all optical measurement systems is shown in Fig. 4. The two PIV cameras are positioned below the rotor. The PIV laser with the laser optics is found far downstream of the rotor. The wind tunnel nozzle is marked with a white box upstream of the rotor. On both sides of the nozzle, a BOS camera is placed. On the right side of Fig. 4, the blade tip camera can be seen.

\subsubsection{Background oriented schlieren (BOS)}

BOS is a method to visualize and measure density gradients in transparent and compressible media. The method is comparable to techniques such as shadowgraphy or classic schlieren measurements (Settles 2006). According to the Gladstone-Dale relation (Liepmann 2013), a change in the density of a gas leads to a change in the refractive index, which then leads to a deflection of light rays.

The BOS method is based on the work of Dalziel et al. (2000) and was first introduced in 1999 by Meier (1999) and in 2000 by Raffel et al. (2000a, 2000b) as well as Richard et al. (2000). BOS requires a suitable background, a camera,

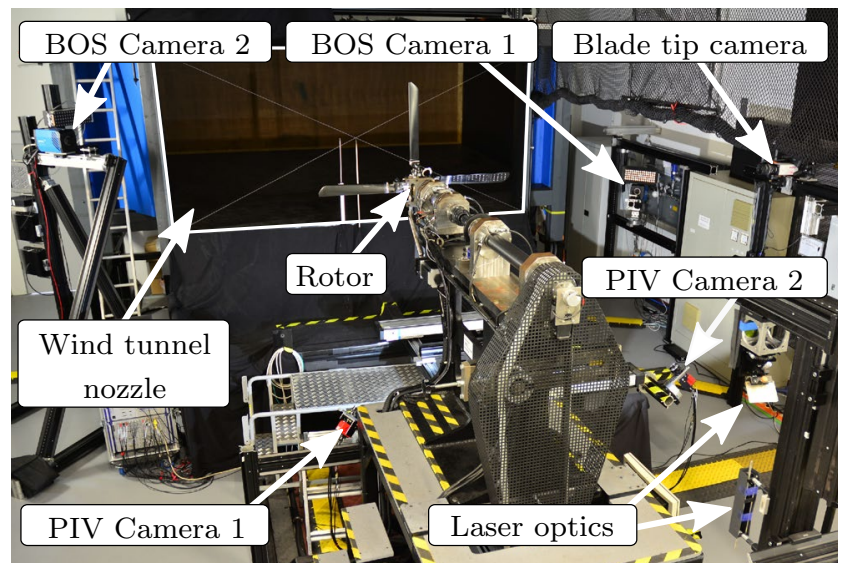

Fig. 4 Overview of the RTG with all optical measurement systems 


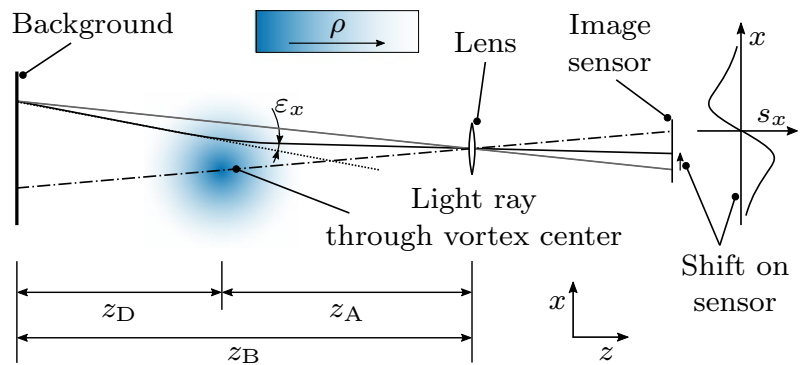

Fig. 5 Schematic of the BOS shift in $x$-direction $s_{x}$ resulting from the density field of a vortex

and a light source. An overview of the BOS technique is given by Raffel (2015). The feasibility of using BOS data for a 3D-reconstruction of vortex filaments was demonstrated, for example, on a full-scale helicopter rotor by Schairer et al. (2013).

The pinhole model of a typical BOS setup is shown in Fig. 5. The region of reduced density (in this case a vortex) is located between the background plane and the camera (illustrated as a lens and an image sensor). The background plane features a pattern of random dots.

The change in the refractive index deflects light rays coming from the background by an angle $\varepsilon_{x}$. The deflection, in combination with the geometry of the BOS setup, leads to an apparent shift $s_{x}$ of the background pattern on the image sensor:

$s_{x}=f\left(\frac{z_{\mathrm{D}}}{z_{\mathrm{D}}+z_{\mathrm{A}}-f}\right) \varepsilon_{x}=S \varepsilon_{x}$.

The influence of the geometry can be summed up by the sensitivity factor $S$.

The comparison of a recording without a vortex (solid gray line in Fig. 5) and one with a vortex (solid black line) results in an apparent shift on the sensor. The light ray is deflected in the direction of higher density. Following the geometry depicted in Fig. 5, a shift toward the vortex center is present on the image sensor. The resulting shift distribution is shown on the right in Fig. 5. The light ray passing through the center of the vortex is not deflected since no gradients perpendicular to its path occur. The apparent shift in the measurement images can be computed by a correlation of each image with an undisturbed reference image of the background.

In comparison to PIV, it is relatively simple to measure the shape and the dynamics of a vortex thread in a 3D measurement volume with BOS. To achieve a reconstruction of the $3 \mathrm{D}$ vortex positions, a stereoscopic setup is needed. The BOS system used for the current investigation consisted of two PCO.dimax 54 CMOS cameras and lenses with $f=300 \mathrm{~mm}$, and $f_{\#}=32$. Two HardSoft MultiLED arrays of light emitting diodes (LED) were used to illuminate the background plane and obtain a sufficient contrast for the measurement. The two background planes were equipped with retro-reflective foils with printed black dots of $1 \mathrm{~mm}$ diameter. The LED arrays were positioned close to the cameras to maximize the signal intensity.

The dimensions of the BOS setup $\left(z_{\mathrm{A}}\right.$ and $\left.z_{\mathrm{D}}\right)$, the resulting spacial resolution in $\mathrm{px} / \mathrm{mm}$, the size of the FOV as well as the sensitivity $S$ according to Eq. (2) are given in Table 2 for both cameras.

The apparent shift on the image senor was determined by a cross-correlation with the commercial software DaVis by LaVision, the same software also used for the PIV evaluation. The evaluation of the data was performed with an initial window size of $32 \times 32 \mathrm{px}$ without a weighting function and an overlap of $50 \%$. The final passes had a window size of $12 \times 12 \mathrm{px}$, a round weighting function, and an overlap of $75 \%$. The obtained values of the apparent shift are well below $1 \mathrm{px}$.

Besides the 3D reconstruction of vortex positions, a second objective of the current investigation is to obtain data on the density distribution over the vortex radius. Hence, the spatial resolution of the BOS system is important. The physical size of the final window is $1.71 \mathrm{~mm}$, which is about half the smallest measured vortex core radius. Therefore, a low-pass filtering effect on the shift data is expected.

When aiming at the interpretation of the shift distribution over the vortex radius, the image blur of the recordings also has to be considered. The background plane must be in focus since the image of the background pattern is used for the correlation to obtain the apparent shift. This results in a blurred image of the density object, if the depth of field of the camera is smaller than the distance $z_{D}$, which is usually the case. A measure for the image blur is the circle of confusion (CoC, Ref. (Greenleaf 1950)), which describes the diameter of the blur spot on the image sensor created by a point source. The $\mathrm{CoC}$ can be calculated by

$\mathrm{CoC}=\frac{1}{f_{\#}} \frac{f^{2} z_{\mathrm{D}}}{z_{\mathrm{A}}\left(z_{\mathrm{A}}+z_{\mathrm{D}}-f\right)}=\frac{S}{f_{\#}} \frac{L_{\text {sensor }}}{L_{\mathrm{FOV}}+L_{\text {sensor }}}$.

Following Eq. (3), the $\mathrm{CoC}$ on the camera sensor of the current setup can be calculated as $0.389 \mathrm{~mm}$ for Camera 1 and $0.423 \mathrm{~mm}$ for Camera 2. This translates to $4.9 \mathrm{~mm}$ and $4.6 \mathrm{~mm}$ in the respective object planes.

Table 2 Geometric parameters of the BOS setup

\begin{tabular}{llllll}
\hline & $\begin{array}{l}z_{\mathrm{A}} \\
(\mathrm{mm})\end{array}$ & $\begin{array}{l}z_{\mathrm{D}} \\
(\mathrm{mm})\end{array}$ & $\begin{array}{l}\mathrm{px} / \mathrm{mm} \\
(-)\end{array}$ & $\begin{array}{l}L_{\mathrm{FOV}} \\
(\mathrm{mm})\end{array}$ & $\begin{array}{l}S \\
(\mathrm{~mm})\end{array}$ \\
\hline Camera 1 & 4660 & 4474 & 7.2 & 279 & 169 \\
Camera 2 & 4064 & 4342 & 8.3 & 243 & 162 \\
\hline
\end{tabular}


In addition to the calculation of the resolution limit based on the $\mathrm{CoC}$, a quantification of the resolution limit due to the image blur, based on recordings of the 1951 USAF resolution test chart, has been performed. The chart consists of groups of black bars of different sizes and distances on a white background. Due to the limited resolution of an optical system, the contrast between the black bars and the white spacing decreases with decreasing bar width. A sample recording of two groups of bars at different sizes is depicted in Fig. 6. The orange dashed line indicates the extraction of horizontal intensity profiles.

Based on Eq. (4), the Michelson contrast can be calculated for every bar group.

Contrast $=\frac{I_{\max }-I_{\min }}{I_{\max }+I_{\min }}$

The physical bar widths of the USAF test chart are corrected for the different viewing angles of both cameras. The calculated values of the Michelson contrast [Eq. (4)] of the four smallest resolvable bar groups for each camera in horizontal and vertical direction are plotted against the bar width in Fig. 7.

The figure shows the decreasing trend of the contrast with decreasing bar widths. Comparable figures often show the line pairs per millimeter on the $x$-axis and are called modulation transfer functions. Based on different contrast thresholds, the minimum resolvable feature size can be estimated [compare, e.g., criteria by Rayleigh and Sparrow (Lasch and Naumann 2006)]. In Fig. 7, a linear fit is used to determine the bar width that leads to zero contrast. It is reached at $3.2 \mathrm{~mm}$ for Camera 1 and at $2.6 \mathrm{~mm}$ for Camera 2. The minimum resolvable feature size is expected above these values and, thus, ranges around the vortex core radii of the current investigation. Compared to the calculated $\mathrm{CoC}$, the minimum resolvable feature sizes are found between $\mathrm{CoC} / 2$ and $\mathrm{CoC}$, depending on the contrast level defining the resolution limit. Since the size of the final interrogation window of the correlation (1.71 $\mathrm{mm}$ in the object plane) is smaller than the determined minima, the driving mechanism that limits
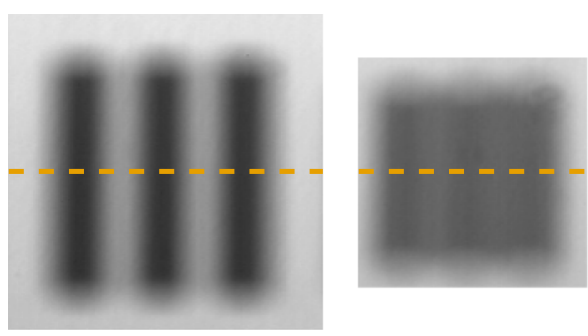

Fig. 6 USAF 1951 resolution test chart: Sample recordings of two groups of different bar widths (Orange dashed line indicates extraction of horizontal intensity profiles)

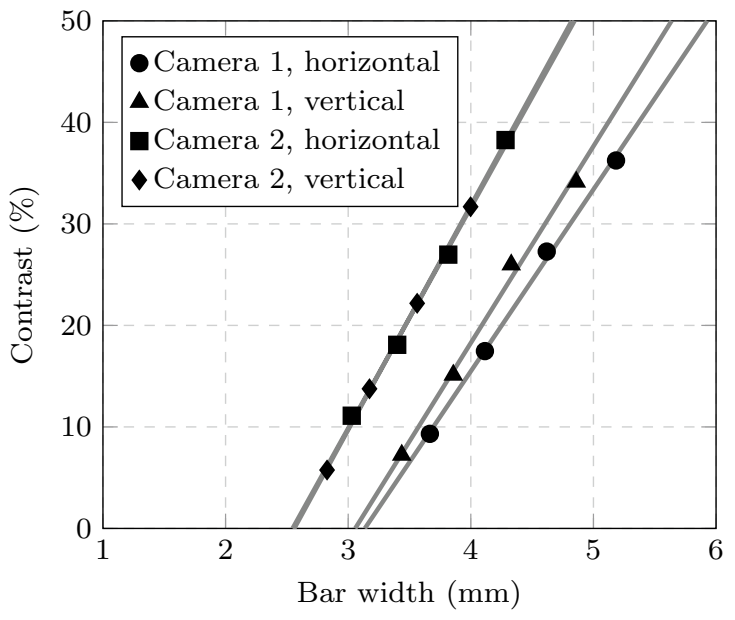

Fig. 7 Modulation transfer function of both BOS cameras in horizontal and vertical direction

the spatial resolution of the currently used BOS system is expected to be the image blur.

A sample BOS result of Camera 2 is shown in Fig. 8, denoting the $x$-gradient of the $x$-displacement $\mathrm{d} s_{x} / \mathrm{d} x$. The sample image is taken from Camera 2, which is positioned on the left side of the nozzle, as seen in Fig. 4. The axial inflow enters the image frame from the left side, and the rotor is located on the right side outside of the image. The tip of the rotor head can be seen as masked-out region in the lower right corner of the image in Fig. 8. The blade tips enter the FOV from the top in a downward motion.

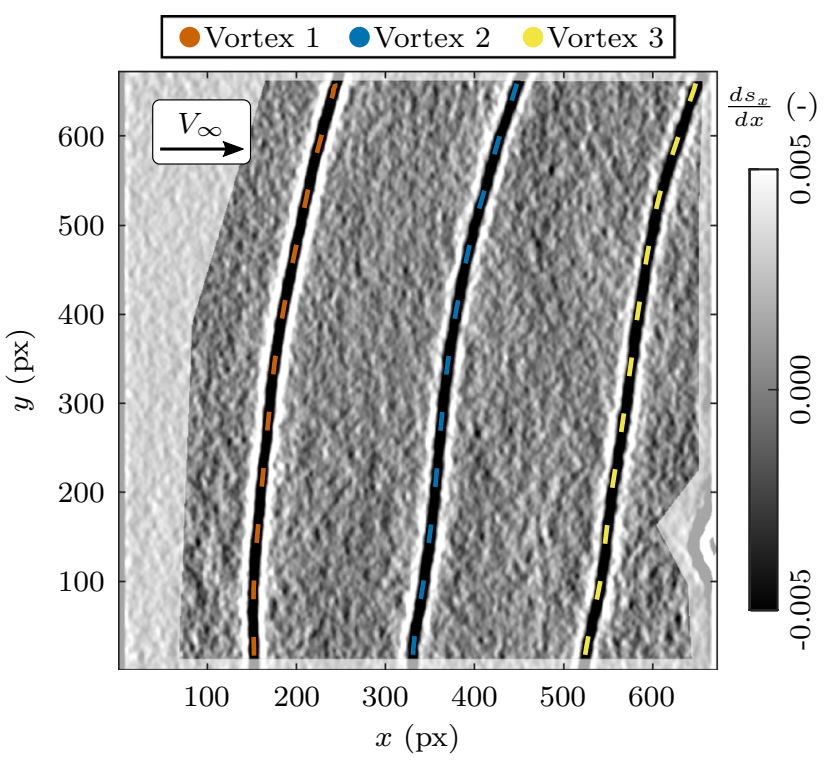

Fig. 8 Sample distribution of the $x$-gradient of the $x$-shift measured by BOS. Three distinct vortices marked by colored dots representing the detected vortex locations 
Three distinct vortices are visible in the FOV. Vortex 1 is the youngest, and vortices 2 and 3 are older by multiples of $90^{\circ}$, as they were produced by preceding blades. Colored dots represent the detected vortex center locations, that were found with the help of NeuronJ by Meijering et al. (2004). The tool was initially designed for the detection of neurites in fluorescence images. It derives a cost function for each pixel, stating the likelihood of belonging to a neurite. A Dijkstra shortest-path algorithm is then used to define the tracings based on predefined track points and the cost function. The similarity of this application to the tracing of blade tip vortices has been shown by Overmeyer (2015), who successfully traced vortices in shift images with different signal-to-noise ratios. The 2D vortex locations of both BOS cameras are further used for a reconstruction of the 3D vortex positions. The 3D reconstruction is carried out based on a calibration of both cameras using a pinhole model. The 3D coordinates of the vortices are then calculated according to epipolar geometry (Debernardis 2020). Each 2D vortex location within the FOV is captured by the two cameras of the stereo setup. The projection of each point onto the image sensor of Camera 1 is represented by a line in Camera 2the epipolar line. The intersection of these lines with the images of the points in Camera 2 then enables the reconstruction of the $3 \mathrm{D}$ location of the vortex center.

\subsubsection{Particle image velocimetry (PIV)}

A stereoscopic PIV system was used to obtain the velocity fields of the vortices evolving from the RTG rotor. Two PCO.edge 5.5 sCMOS cameras were calibrated on a horizontal measurement plane, downstream of the rotor at the blades' trailing edges. The chosen plane captures rotor blade tip vortices whose axes are approximately perpendicular to the measurement plane. Both cameras were equipped with lenses of $f=180 \mathrm{~mm}$, and $f_{\#}=2.8$. The resolution of the cameras is $2560 \mathrm{px}$ in stream-wise, and $2160 \mathrm{px}$ in radial direction. This setup yielded a FOV of $150 \mathrm{~mm} \times 130 \mathrm{~mm}$ with an average spatial resolution of $16.8 \mathrm{px} / \mathrm{mm}$. A Litron Nano L 200-15 PIV low-speed laser with a wavelength of $532 \mathrm{~nm}$ and an energy of $200 \mathrm{~mJ}$ per pulse was used to illuminate the measurement plane. The separation time of the two laser pulses was set to $\Delta t=26 \mu \mathrm{s}$. The width of the light sheet was adjusted to approximately $4 \mathrm{~mm}$. Aerosolized Di-Ethylhexyl-Sebacate (DEHS) oil was used to produce seeding with a size of less than $1 \mu \mathrm{m}$. The seeding generator was positioned close to the inlet of the wind tunnel. This position was found to result in a homogeneous distribution of the particles in the inflow.

The particle images were evaluated by cross-correlation using the commercial software DaVis 8.4 by LaVision. The images were preprocessed using a sliding background subtraction and a particle intensity normalization in sliding windows of $5 \times 5 \mathrm{px}$ to account for inhomogeneities in the laser light sheet and a varying particle intensity.

The correlation was performed iteratively with decreasing window sizes. A window size of $128 \times 128 \mathrm{px}$, an adaptive window shape (Wieneke and Pfeiffer 2010), and an overlap of $50 \%$ were chosen for the initial pass. The final pass also had an adaptive window shape but a window size of $16 \times 16 \mathrm{px}(0.95 \mathrm{~mm}$ in the image plane), and an overlap of $75 \%$. This setting yields 8 to 33 velocity vectors over the vortex core radius (depending on the vortex size). No additional filtering of the results was done in DaVis.

A sample individual PIV result is shown in Fig. 9. The velocity field is one of the 200 instantaneous results of a test case in the upstroke $(t / T=0.26)$ at a vortex age of $\Psi_{\mathrm{v}}=25^{\circ}$. The vortex age $\Psi_{\mathrm{v}}$ is defined as the angular distance between the measurement plane and the trailing edge of the respective blade during acquisition. The data are plotted in the vortex coordinate system, meaning that the vortex core is located at $r=z=0$. The image shows the absolute in-plane velocity as a gray scale distribution. Additionally, every 25 th velocity vector is plotted.

In the area of the vortex core, a lack of particles (particle void) occurs due to the centrifugal forces and the higher density of the seeding particles compared to air. Data in the region of the void are considered unreliable and omitted during further evaluations of the vector fields. The extent of the particle void in each recording is estimated based on the intensity distribution of the particle images. The images are spatially low-pass filtered to eliminate the influence of individual particles and measurement noise, thereby concentrating only on the large-scale particle void. Afterward, the minimum of each image is set to zero by subtraction. The

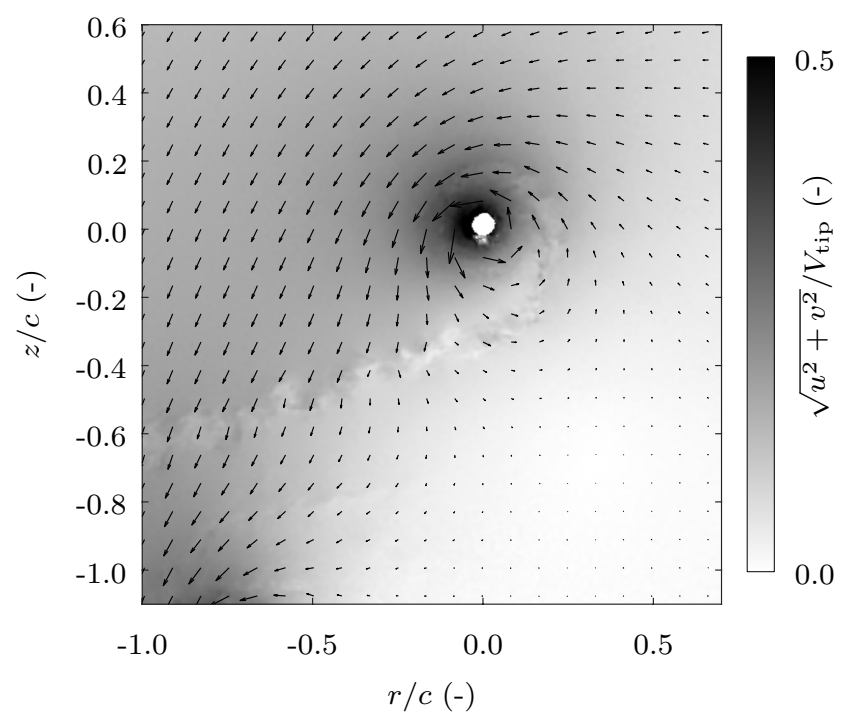

Fig. 9 Sample distribution of the absolute in-plane velocity with overlayed velocity vectors (sub sampled by a factor of 25 ) 
region of the particle void is then defined as the area with an intensity lower than $30 \%$ of the median intensity in each individual image.

The vortex centers are detected based on the filtered distribution of the out-of-plane vorticity. To account for missing information in the vortex center due to the particle void and to suppress the influence of small-scale turbulence, e.g., resulting from the blades shear layer, the vorticity is filtered by a convolution with a normal distribution with the size of an expected vortex. This method was described by van der Wall and Richard (2006) and successfully used by Bauknecht et al. (2017). Small vorticity peaks in the area of the blade shear layer are dampened as a result of the filtering, which increases the robustness and the liability of the vortex center detection. The final vortex center is defined as the centroid of the vorticity distribution in the area with a vorticity greater than $90 \%$ of the maximum. Previous investigations have shown that this approach offers a reliable center detection despite dealing with noisy data (Bauknecht et al. 2015). Also, Bhagwat and Ramasamy (2012) found methods relying on an area of measured data (as opposed to only using a few points in the vortex core) to be more reliable.

Based on the vortex center, the swirl velocity $V_{\theta}$ as well as the circulation $\Gamma$ can be calculated. This is done by applying a radial binning around the detected vortex center. Radial bins corresponding to the spatial resolution of the PIV data are defined. For each bin, the mean swirl velocity of all grid points within the bin is calculated. This approach, which was also used by Wolf et al. (2019) and Braukmann et al. (2020), assumes rotationally symmetric vortices. Using the swirl velocity of each bin with a certain radius, the circulation can be derived as the line integral of the swirl velocity.

\subsubsection{Combining BOS and PIV}

The FOV of the BOS system in relation to the rotor is shown as a blue volume in Fig. 10. Furthermore, the figure shows the PIV plane as a green area. For each measurement, the PIV system provides information at one specific vortex age $\Psi_{\mathrm{v}}$ only, while the BOS system captures vortex ages between $\Delta \Psi_{\mathrm{v}}=-8^{\circ}$ and $15^{\circ}$ off the vortex age measured with PIV.

The captured vortex age is defined by the delay between the reference blade passing the measurement plane of PIV and the time of acquisition. The drive shaft of the RTG is equipped with a rotational encoder, which provides a signal with one pulse per revolution (1/rev) and a signal with 1000 pulses per revolution (1000/rev), allowing for the exact timing of, e.g., cameras and light sources. Both the BOS and the PIV system were synchronized using the encoder signals. This approach makes it possible to capture the vortices' velocity and density distributions at the same time. At the position of the PIV plane, the data on density can be combined with the data on velocity.

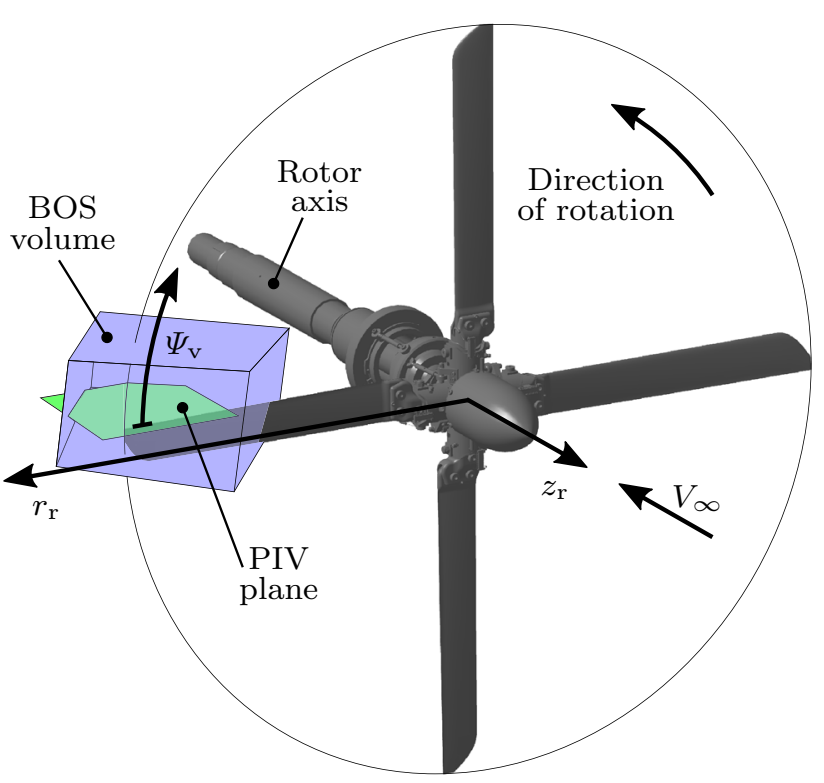

Fig. 10 Measurement volume of BOS and PIV plane in relation to the rotor

\subsection{CFD simulation}

The CFD simulations are taken from Goerttler et al. (2020) and were performed in the department of helicopter aerodynamics at the DLR Göttingen. The numerical computations were performed using DLR's finite volume solver TAU. Four identical grids with 9.5 million points have been used for the blades. The blade grids are embedded in a background grid with a total size of 48 million points. The first $80^{\circ}$ of one blade tip vortex is refined with a curved, structured region, following the expected path of the blade-tip vortex. The normal spacing to the vortex core propagation direction is $s_{\mathrm{c}} / c=0.0034$ at a very young wake age and increases up to $s_{\mathrm{c}} / c=0.0091$ at a vortex age of $\Psi_{\mathrm{v}}=90^{\circ}$. A zonal approach with a large-eddy simulations (LES) model (Travin et al. 2002) is employed on the four-bladed configuration to reduce numerical dissipation. The LES model is used around the fine structured grid, in all other regions, the Menter shear-stress transport (SST) model (Menter 1993) is used. Therefore, smaller scales which trigger viscous dissipation are not modeled but resolved. This leads to less dissipating and more accurate solutions than pure Reynolds-Averaged Navier Stokes (RANS) approaches (Goerttler et al. 2020). The chimera method is used to carry out the data exchange between the pitching blade grids and the background grid. The results of the computations were published by Goerttler et al. (2020) and are a valuable addition to a discussion of the experimental data presented in this paper. 


\section{Results and discussion}

PIV allows a quantification of the vortex strength by means of the vortex circulation. It is common to use the total circulation $\Gamma_{\infty}$ for quantitative comparisons. Due to the limited FOV of the PIV system, the circulation at a fixed radial position in the vortex $r=0.5 c$ is chosen as a measure for the vortex strength. Even with a much larger FOV, it is not possible to derive a reliable value for $\Gamma_{\infty}$ e.g., due to the influence of the connected shear layer from the rotor blades (Goerttler et al. 2020). The use of the circulation at $\Gamma_{0.5 c}$ for a quantitative interpretation was successfully demonstrated by Wolf et al. (2019) and Braukmann et al. (2020) during a comparison of different PIV measurements and also the comparison of experimental results with numerical computations.

Figure 12 shows the vortex circulation $\Gamma_{0.5 c}$ for different vortex ages $\Psi_{\mathrm{v}}$ over the pitch angle of the blade at the time of vortex creation, obtained by PIV. The circulation is calculated based on a conditional average of the 200 instantaneous velocity distributions for each test case: Before averaging, the vorticity distributions are aligned according to the detected vortex centers (van der Wall and Richard 2006). Furthermore, a radial binning, assuming rotationally symmetric vortices, is used.

The circulation measured by PIV shows the expected trend with a varying pitch angle. Between up- and downstroke, a hysteresis is present. The different vortex ages do not have a visible effect up to the maximum age measured by PIV $\left(\Psi_{\mathrm{v}}=70^{\circ}\right)$. The vortex circulation resulting from the chosen cyclic pitch setting in the current case varies by a factor of three. This change in the vortex strength is one of the main influences on the evolving rotor wake.

\subsection{D vortex system}

A reconstructed snapshot of the azimuth-dependent wake of the RTG rotor under cyclic pitch conditions is shown in Fig. 11. A 3D model of the rotor is added to ease the interpretation of the downwash structure. The four characteristic phases of the blade pitch cycle $(t / T=0.00,0.25,0.50$, and 0.75$)$ are marked in the four corners of the image. Each of the four blades is located $25^{\circ}$ behind one of these phases. Behind each blade, a vortex is present, which was produced at the pitch angles stated around the structure. As a rule of thumb, the vortices on the left side of Fig. 11 were shed from the blades during upstroke and the ones on the right side during the downstroke.

The vortices are represented by averaged and convolution filtered out-of-plane vorticity distributions from PIV,

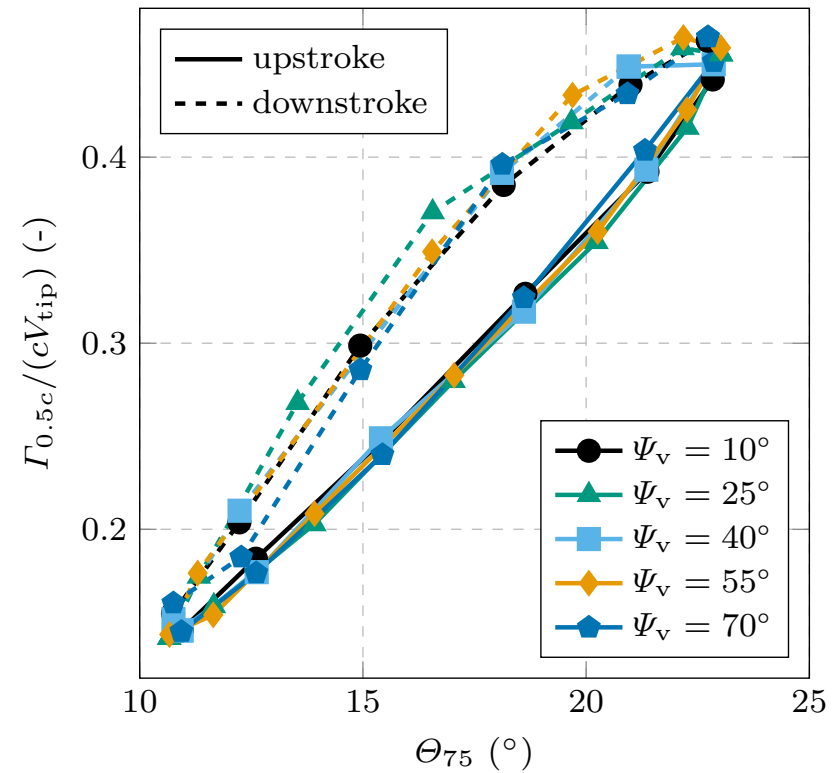

Fig. 11 Vortex circulation $\Gamma_{0.5 c}$ at $r=0.5 c$ at different vortex ages $\Psi_{\mathrm{v}}$ over the pitch angle

in all 20 measurement planes. For a better visibility, values close to zero (between -0.035 and 0.035 ) are set to transparent. The vortices in the PIV planes are linked by colored dots, which state the average center position of the vortices as derived from the BOS data. The dots are colored with respect to the blade of origin of each vortex.

The BOS-detected vortex centers are matched by circular spots of positive vorticity, as seen by PIV. The tip vortices are fed by the blade shear layers, which also appear in the vorticity distribution as linearly elongated structures pointing toward the hub. In some cases, the shear layer's sense of rotation changes further inboard, marked by a transition from red to blue coloring. This point marks the local maximum in the radial lift distribution. Similar findings were described by Milluzzo and Leishman (2016).

Depending on the pitch phase, one or two vortices are present in the FOV of PIV. In cases with a low pitch angle, the downwash velocity is reduced, which leads to a reduced convection rate of the vortices and, thus, two vortices inside the limited FOV of PIV. The FOV of the BOS system on the other hand contains one to three vortices, leading to lines that continue below the vorticity planes from PIV. The oldest vortex shown in the snapshot of Fig. 11 and captured by BOS is around $200^{\circ}$ old and evolved from Blade 4 . The reconstructed overview of the vortex system provides a good impression of the asymmetry and azimuthal dependency of the captured rotor wake resulting from the cyclic pitch setting. 
Fig. 12 Overview of the PIV planes (normalized out-of-plane vorticity color coded, schematic of rotor for reference). The lines linking the planes represent the $3 \mathrm{D}$ vortex locations reconstructed from the BOS measurements

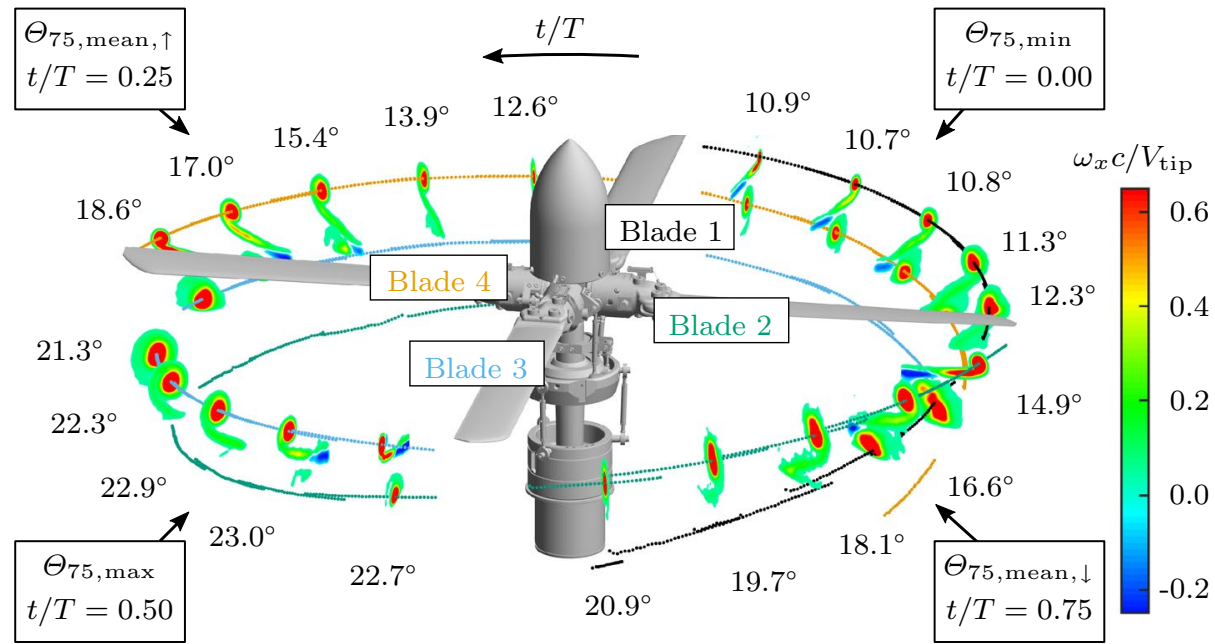

(a)

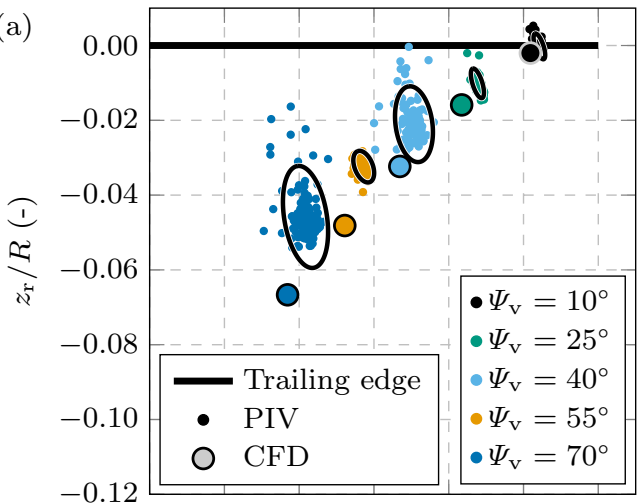

(b)

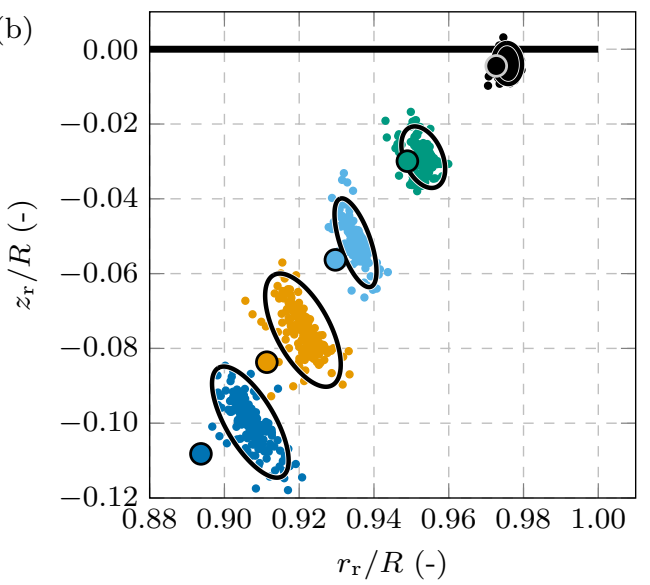

Fig. 13 Individual vortex locations by PIV with ellipses representing a confidence interval of $95 \%$ and positions from CFD calculations. Vortex shed at the minimum pitch angle $(t / T=0.00)$ (a) and at the maximum pitch angle $(t / T=0.51)(\mathbf{b})$, both at different vortex ages

\subsection{Vortex locations and aperiodic wandering}

Figure 13 shows the vortex locations obtained by PIV, shed by a blade at the minimum pitch angle at $t / T=0.00$ (Fig. 13a) and at the maximum pitch angle at $t / T=0.51$
(Fig. 13b). Both figures show the locations at different vortex ages $\Psi_{\mathrm{v}}$. The trailing edge of the blade is included as black line at $z_{\mathrm{r}} / R=0$. The colored dots represent the 200 individual center locations from the PIV measurement. The locations are corrected for the blade bending based on the data from the blade tip camera.

The ellipse around each group of locations represents a confidence interval of $95 \%$ (two times the standard deviation) of the individual results. It can be seen that the scatter of the vortex locations has a dominant axis for all cases. This major axis appears to be approximately perpendicular to the mean convection trajectory. This finding hints at a variation of the individual trajectories rather than a change in the convection velocity along a steady trajectory. Similar results were discussed by Mula et al. (2011), who also found increasing scatter ellipses with rising vortex age. The cases at $\Psi_{\mathrm{v}}=25^{\circ}$ and $\Psi_{\mathrm{v}}=55^{\circ}$ in Fig. $13 \mathrm{a}$ shows an exception to this trend, although the reason for this finding remains unknown.

By comparing Fig. 13a and b, the influence of the blade pitch can be studied. At the minimum pitch angle, the downwash velocity is smaller compared to the case with maximum pitch, leading to a reduced convection rate of the tip vortices. Also, the aperiodic scattering is dependent on the pitch angle.

In addition to the PIV results, the locations computed by the CFD simulation are shown as colored spots. The computations and the experiment show comparable results regarding the convection direction and the influence of the pitch angle. The convection rate in the CFD is higher compared to the PIV results.

\subsection{Position estimation by BOS and PIV}

The vortex scatter shown in Fig. 13 is based on the PIV data. The same values can be obtained from the $3 \mathrm{D}$ 


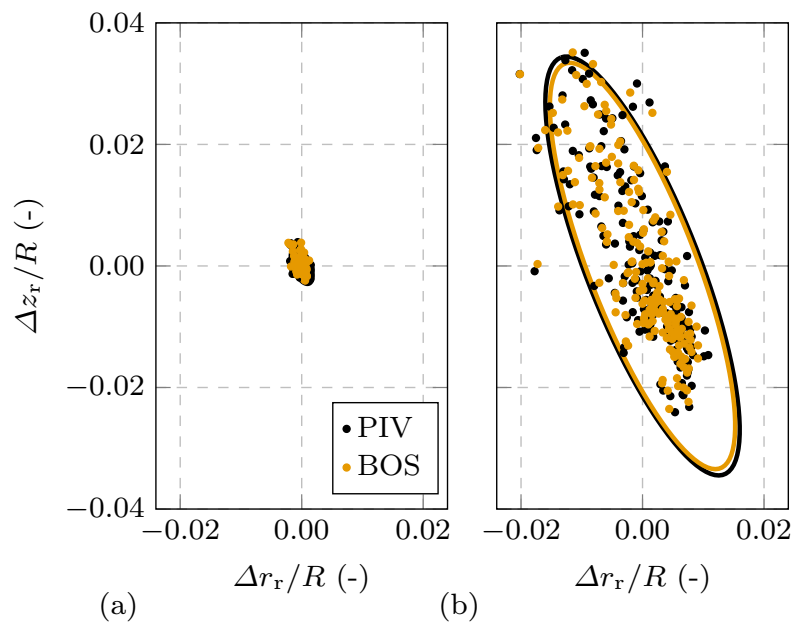

Fig. 14 Scatter of vortex locations for two sample cases obtained by PIV and BOS. $\Theta_{75}=16.2^{\circ}, \Psi_{\mathrm{v}}=10^{\circ}$, during the upstroke (a) and $\Theta_{75}=17.3^{\circ}, \Psi_{V}=55^{\circ}$, during the downstroke (b)

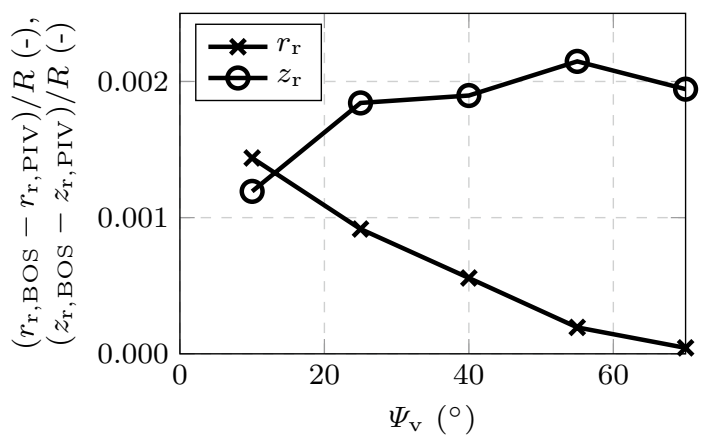

Fig. 15 Absolute difference between mean vortex locations by PIV and BOS in $r_{\mathrm{r}}$ - and $z_{\mathrm{r}}$-direction over the vortex age $\Psi_{\mathrm{v}}$ for a sample case during upstroke (Blade $4, t / T=0.30-0.13$ )

vortex positions from BOS. The vortex locations from BOS, extracted at the PIV plane, as well as the comparable values from PIV are plotted in Fig. 14 for two sample cases with very different scatter intensities, both at the same scale. To focus on the representation of the aperiodic vortex wandering, the mean position of each group was subtracted from the individual positions. This step removes a constant offset between the different techniques, which will be discussed in Fig. 15. The comparison of Fig. 14a, b gives an impression of the range of scatter observed: The two major scatter axes vary by a factor of ten within the data captured in the current investigation.

Comparing the results from PIV and BOS in Fig. 14b, a good agreement between the individual vortex locations estimated with two very different techniques is evident. The individual positions and, as a result, also the scatter ellipses agree well. The agreement can be quantified by the correlation coefficient of the PIV and BOS positions, which calculates to 0.998 for the case shown in Fig. 14b. The correlation for the case in Fig. 14a is 0.855 , which is significantly smaller. This finding can be explained by two causes: (1) In case of a small scatter, the error between PIV and BOS (e.g., due to noise) is relatively large, which leads to a decrease in the correlation. (2) In case of the small scatter in Fig. 14a, the resolution limit of the BOS technique is reached. Since the vortex positions in the gradient of the shift are found using only the accuracy of integer pixel, the resolution limit in the BOS results leads to a stepping in the BOS positions. The step size can be approximated to be $7.5 \cdot 10^{-4} R(\approx 0.5 \mathrm{~mm})$ in the case presented in Fig. $14 \mathrm{a}$, which is $1 / 8$ of the major axis length of the resulting scatter ellipse.

Figure 15 shows the absolute difference between the mean vortex locations obtained by PIV and by BOS. The two graphs represent the differences in $r_{\mathrm{r}}$ - and $z_{\mathrm{r}}$-direction over the vortex age for a sample case during the upstroke (shed from Blade $4, t / T=0.30-0.13$ ). With rising vortex age, the offset in radial direction $\left(r_{\mathrm{r}}\right)$ reduces to zero, while the offset in $z_{\mathrm{r}}$-direction increases. The variation of the offsets with the vortex age can be ascribed to a misalignment between the individual calibrations of both systems. The absolute difference between PIV and BOS is always below $0.0023 R\left(1.5 \mathrm{~mm}\right.$, or $\left.\approx 0.5 r_{\mathrm{c}}\right)$ for the shown cases. The agreement of both techniques, despite the different approaches and sources of error, is satisfactory and sufficient for the interpretation of the vortex system presented in this paper.

\subsection{Phase dependency of the rotor wake}

Figure 16 quantifies the rotor wake given in Fig. 11 over the pitch phase $t / T$. Figure 16a and $b$ states the pitch angle at the vortex origin $\Theta_{75}$ and the vortex age at the recording of the vortex $\Psi_{v}$, respectively. These quantities ease the interpretation of the results in Fig. 16c-f. The combination of vortex age and pitch phase correspond to moving along a vortex up to an age of $70^{\circ}$ in Fig. 11. The data in both figures are colored with respect to the blade of origin of each vortex.

The normalized vortex circulation $\Gamma_{0.5 c}$ is given in Fig. 16c. The values follow the expected trend according to the changing pitch angle over the phase. The circulation changes by a factor of three during the pitch cycle. The hysteresis between up- and downstroke observed in Fig. 12 is present as horizontal offset between the curve of the circulation and the pitch angle in Fig. 16a.

Figure $16 \mathrm{~d}$ shows the length of the major axis of the scatter ellipses $a$ introduced in Fig. 13, normalized with the rotor radius $R$. The values obtained by both PIV and BOS are given. The data indicate a strong influence of the vortex age on the aperiodic vortex scatter. This trend is superimposed by an influence of the pitch phase. The major scatter length 


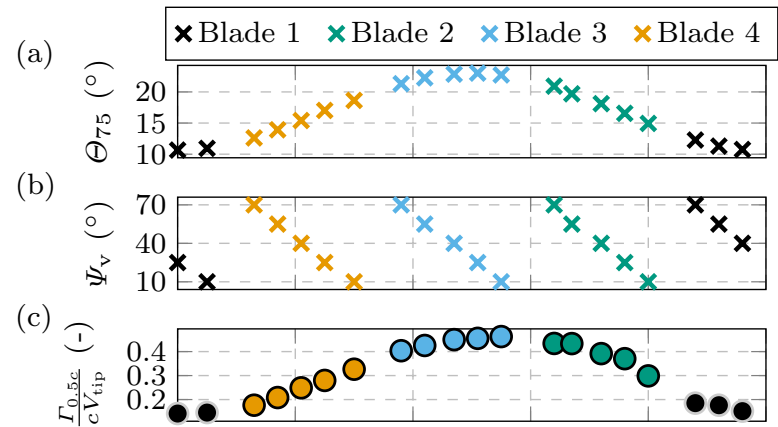

(d)

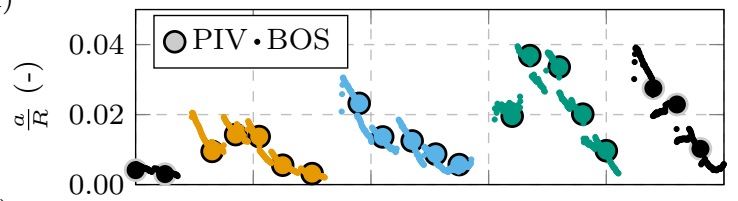

(e)
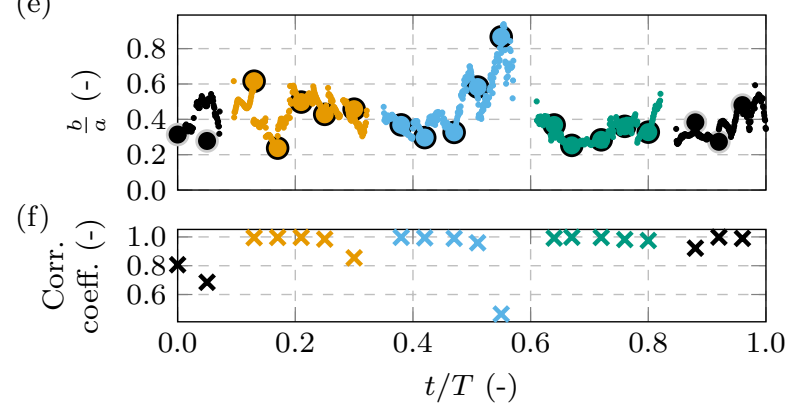

Fig. 16 Several parameters characterizing the rotor wake plotted over the pitch phase $t / T$

increases faster during the downstroke motion of the blades $(t / T>0.5)$, hinting at an increased sensitivity to perturbations as also found by a previous investigation at the RTG (Braukmann et al. 2020). A good agreement between PIV and BOS can be seen during the entire pitch cycle.

The aspect ratio of the scatter ellipses is plotted in Fig. 16e. For $b / a=1$, the confidence region is perfectly circular, whereas for smaller values, the major axis dominates and the ellipse is elongated along this axis. For most pitch phases, the aspect ratio is found between 0.2 and 0.6. The young vortex evolving from Blade 3 shows an exception to this trend, with aspect ratios up to 0.9 , indicating a rather circular region of scatter. The vortex shed from Blade 2 during the downstroke, shows a comparably constant aspect ratio of the confidence ellipses, although the major axis length strongly depends on the vortex age and the phase of origin. This states a symmetric growth of the scatter region along the vortex.

The results obtained by PIV and BOS are in good agreement for most comparisons in Figs. 14, 15, and 16d and e. The agreement can be quantified by the Pearson correlation coefficient, which gives a measure of the linear dependence of the 200 individual positions measured with both methods. The resulting values for the vortices of the snapshot shown in Fig. 11 are plotted in Fig. 16f against the pitch phase. The resulting values are above 0.9 for most of the cases. The youngest vortices show a strongly decreased correlation for the vortices shed by Blade 1, 3 and 4 . The reduced correlation for these cases can be explained by the small area of the position scatter for young vortices, as discussed in Fig. 14.

The scatter ellipses obtained by PIV are limited to the 20 discrete measurement planes. Since BOS enables the reconstruction of the vortex position in a measurement volume, it is possible to obtain the scatter ellipses denoting two times the standard deviation of the vortex scatter over the entire rotor azimuth. The resulting structure is shown in Fig. 17. The elliptic tubes are colored with respect to the blade of origin and represent the volume in which the vortex center appears with a confidence of $95 \%$.

Figure 17 clearly shows the growth of the scatter region with increasing vortex age for all four vortices. Additionally to the data plotted in Fig. 16, vortex ages over $\Psi_{\mathrm{v}}=70^{\circ}$ are also represented.
Fig. 17 3D depiction of the scatter ellipses of the vortex center location with a confidence interval of $95 \%$, obtained from the BOS measurements. Vortices color coded with respect to the blade of origin

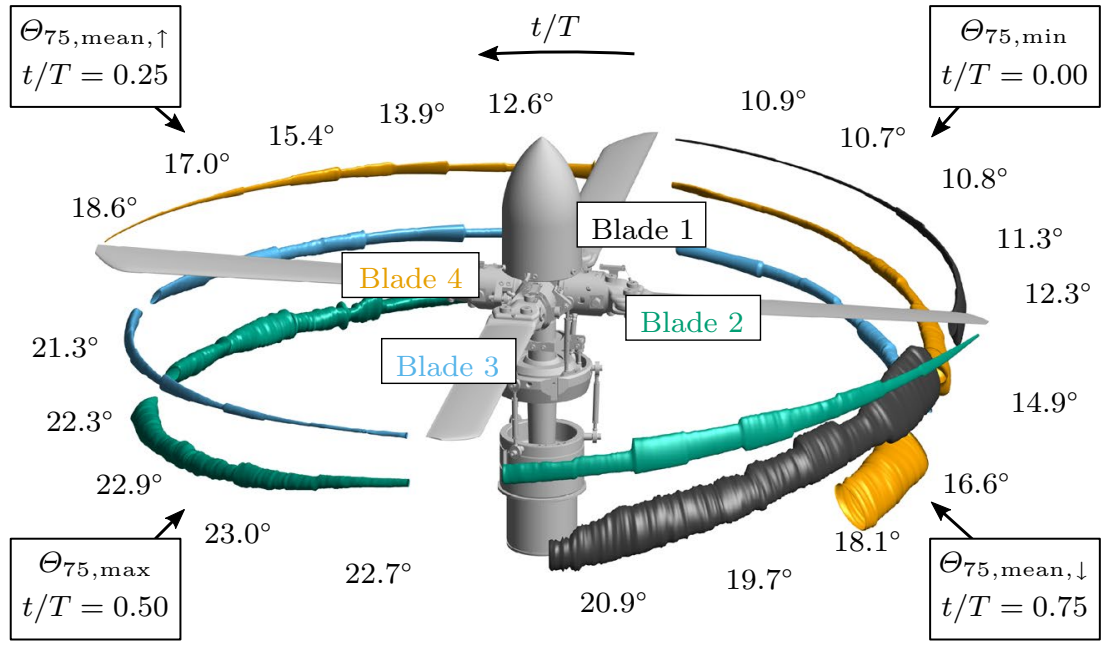


In addition to the vortex growth with rising age, a stepping in the size of the ellipses is apparent. The snapshot in Fig. 17 is the result of 20 individual measurements, that were all performed in the BOS measurement volume shown in Fig. 10. Due to the rectangular shape of the wind tunnel nozzle and the position of the BOS measurement volume, the wind tunnel shear layer has an increased influence on the upper end of the volume. A close wind tunnel shear layer increases the perturbations, which leads to an increase in the vortex scatter and to increased scatter ellipses. Therefore, the scatter within each single measurement increases stronger than expected with rising vortex age. The combination of the individual measurements into one snapshot, thus, leads to the stepping seen in Fig. 17.

Despite this influence, the varying size of the scatter ellipse due to the varying pitch angle during the vortex creation is visible in Fig. 17. Vortices during the downstroke (around $t / T=0.75$ ) show an increased position scatter. This finding hints at an increased instability of the rotor wake during this phase of the pitch cycle. Figure 17 gives a good impression of the extent of the vortex scatter in relation to the rotor. The varying vortex positions affect the vortex-vortex as well as the blade-vortex distances, which is relevant when predicting interactions.

\subsection{Swirl velocity profiles and vortex models}

The PIV data do not provide reliable information in the region of the particle void. A common method to overcome this deficit is the use of analytical vortex models. Vatistas et al. (1991) formulated a generalized vortex model representing the swirl velocity distribution $V_{\theta}$ over the vortex radius $r$ :

$V_{\theta}=V_{\theta, \max } \frac{r}{r_{\mathrm{c}}}\left(\frac{1}{1+\left(\frac{r}{r_{\mathrm{c}}}\right)^{2 n}}\right)^{\frac{1}{n}}$.

Different, previously described vortex models can be obtained by choosing the integer factor $n$ : For $n=1$, the model by Kaufmann (Kaufmann 1962) or Scully (Scully and Sullivan 1972) and for $n=2$, the model by Bagai-Leishman (Bagai and Leishman 1995) is retrieved. The vortex model by Vatistas from 1991 (Eq. (5)) is further referred to as Vatistas1991.

In 2015, Vatistas introduced a modified vortex model, featuring a turbulence factor $\beta$ :

$V_{\theta}=V_{\theta, \max } \frac{r}{r_{\mathrm{c}}}\left(\frac{1+\beta}{1+\beta\left(\frac{r}{r_{\mathrm{c}}}\right)^{2 n}}\right)^{\frac{1+\beta}{2 n \beta}}$.
This new, extended model provides an additional factor, which can be varied during the fit to experimental data. An increased $\beta$ represents a higher turbulence in the vortex and leads to a broadened swirl velocity distribution. Following Vatistas (Vatistas et al. 2015), for $\beta=1$, the swirl velocity distribution of the new model compares to the laminar formulation. The newer model from 2015 is referred to as Vatistas2015 in the following discussion.

The swirl velocity calculated on the basis of the PIV data is plotted in Fig. 18 as black circles. The two figures show the swirl velocity of vortices at a vortex age of $25^{\circ}$ in the up- (Fig. 18a) and downstroke (Fig. 18b). The distribution is taken from one of the 200 instantaneous measurements of that test case. In addition to the experimental data, best fits of the four previously described vortex models (Vatistas 1991 and 2015, each for $n=1$ and $n=2$ ) are plotted. From both figures, it can be seen that it is possible to fit the analytical models to instantaneous swirl velocity distributions, based on only the data outside the particle void.

The variables optimized during the vortex fit are the maximum swirl velocity $V_{\theta \text {, max }}$, the vortex core radius $r_{\mathrm{c}}$, and, in case of the Vatistas 2015 model, also the turbulence factor $\beta$. From the comparison in Fig. 18, it can be seen that the best fit of the Vatistas1991 model underrepresents the swirl velocity at high radii for both choices of $n$. This leads to an underrepresentation of the vortex circulation. The model

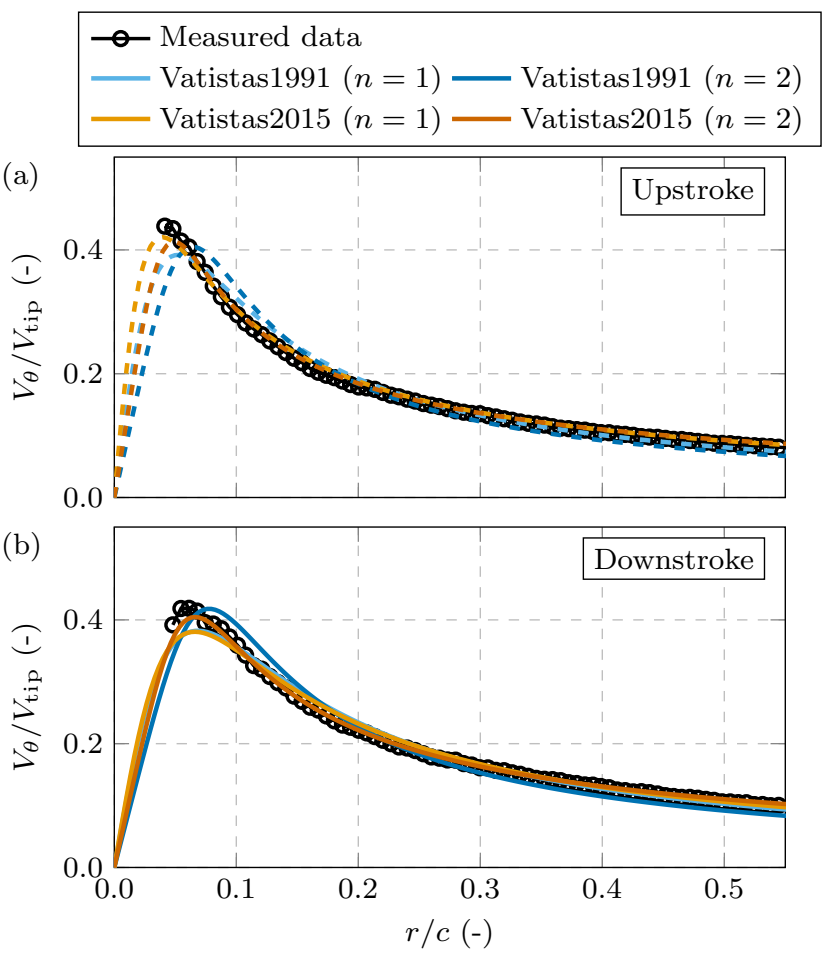

Fig. 18 Sample of fits to instantaneous swirl velocity profiles of a vortex produced by a blade in upstroke $(t / T=0.26)$ (a) and in downstroke $(t / T=0.76)(\mathbf{b})$, both at a vortex age of $\Psi_{\mathrm{v}}=25^{\circ}$ 
also leads to a reduction of $V_{\theta, \max }$ and an increased vortex core radius compared to the PIV data. The best fit of the newer Vatistas 2015 model, featuring the turbulence correction, shows a better agreement with the data of the presented cases. For both the up- and downstroke case and for both factors $n$, the profiles represent the key features more closely to the experiment.

A measure to quantify the goodness of a fit for a range of vortices is the sum of squared residuals. The average value of the 200 instantaneous results for both models, both factors $n$ as well as for two vortex ages in each the up- and downstroke is shown in Fig. 19. It can be seen that the newer Vatistas2015 model leads to lower residuals compared to its predecessor. This can be ascribed to the additional fit parameter representing the turbulence within the vortex. The Vatistas 2015 model with $n=1$ leads to the lowest residuals and is therefore chosen for further discussions of the results.

A previous investigation of blade tip vortices at the RTG by Wolf et al. (Wolf et al. 2019) used a fit to the average profile of multiple PIV images instead of fits to the individual PIV measurements. Furthermore, the current investigation features an increased spatial resolution, leading to an increase in the maximum measured swirl velocities by PIV (Wolf et al. 2019). Wolf et al. compared several vortex models and found the Vatistas2015 model with $n=2$ and $\beta=1.3$ to be the best fit to the obtained swirl velocity profiles at a constant pitch angle of $\Theta_{75}=17.1^{\circ}$ and for several vortex ages up to $325^{\circ}$.

The application of the Vatistas 2015 model to the data of two test cases, both at $\Psi_{\mathrm{v}}=25^{\circ}$ in the up- and downstroke, is shown in Fig. 20. Both vortices were shed from a blade at comparable pitch angles of $\Theta_{75}=17.0^{\circ}$ and $16.6^{\circ}$, respectively. The gray lines represent all 200 individual profiles from PIV. The black line is the respective average. Only data

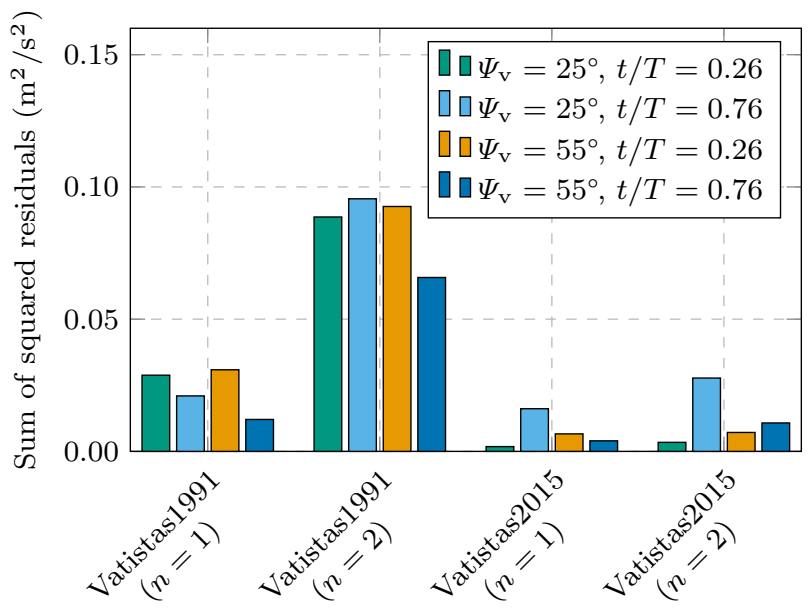

Fig. 19 Sum of squared residuals for four different vortex models and four different test cases outside the particle void are provided. The orange dotted line shows the average of the individual fits of the Vatistas 2015 model with $n=1$.

Despite the strong aperiodicity in the downstroke, the average fit represents both average swirl velocity profiles comparably well (see Fig. 20). This is achieved by different turbulence factors $\beta$. The mean $\beta$ in the upstroke case is 1.25 , whereas the mean $\beta$ in the downstroke case is 1.07 . Contrary to the expected result of a higher turbulence in the downstroke, the fitted $\beta$ suggests a reduced vortex turbulence in the downstroke compared to the upstroke. In addition to the data based on the PIV measurement, the profile obtained by the corresponding CFD simulation is shown as blue graph. In the upstroke, CFD and PIV agree well for radii above $0.5 c$. In the vortex core, CFD underpredicts the PIV swirl velocities. The deviation in the downstroke is more pronounced, with agreement in the core region but an offset outside the vortex core. The resulting vortex circulation $\Gamma_{0.5 c}$ from CFD will show lower values in the downstroke than obtained by PIV.

Figure 21 displays the 200 individual values of the turbulence factor $\beta$ obtained from the fit to the PIV data. Figure 21a provides the values for the upstroke and Fig. 21b for the downstroke, respectively. Each figure provides the values for two different vortex ages, $\Psi_{\mathrm{v}}=25^{\circ}$ and $55^{\circ}$. It can be

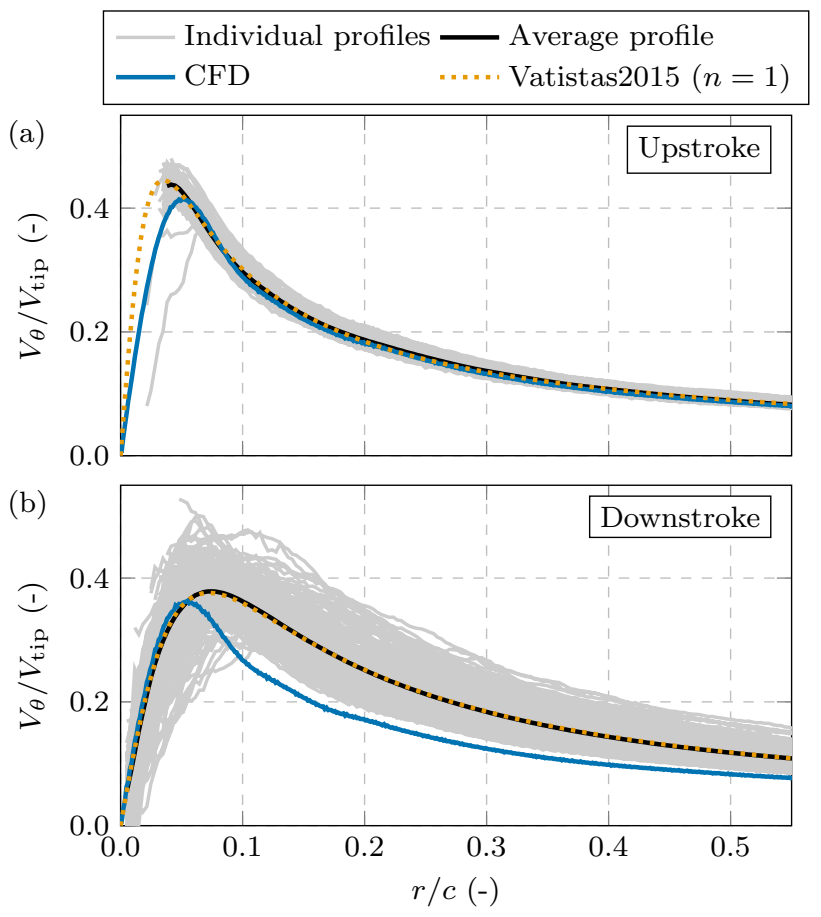

Fig. 20 Normalized swirl velocity over the normalized vortex radius at a vortex age of $\Psi_{\mathrm{v}}=25^{\circ}$. 200 individual and mean distribution from PIV, distribution from CFD, and mean of individual fits to PIV data. Upstroke $(t / T=0.26)$ (a) and downstroke $(t / T=0.76)$ (b) 
seen that the turbulence factor shows only a few occasions of increased deviations from the mean value of $\beta=1.25$.

In the downstroke, the variation is twice as high across the entire 200 measurements, although the mean value is lower than in the upstroke. Thus, a higher aperiodicity in the turbulence factor goes along with a lower turbulence as given by the turbulence factor $\beta$. This is another representation of the finding of a vortex that appears to have a more laminar velocity profile yet being affected by an unsteady downwash topology, as observed in Fig. 20b.

\subsection{Density measurements from PIV and from BOS}

PIV measures the velocity components of a flow. BOS, on the other hand, detects a shift related to the gradient of the density. Bagai and Leishman (Bagai and Leishman 1993) provided a formulation to link these two quantities based on the Navier Stokes equations for an inviscid, stationary, and two-dimensional vortex. Furthermore, the ideal gas law for isentropic flow

$\left(\frac{p}{p_{\infty}}\right)=\left(\frac{\rho}{\rho_{\infty}}\right)^{n_{\mathrm{p}}}$

with $n_{\mathrm{p}}=\gamma=1.4$ was used.

Based on these two assumptions, they stated:
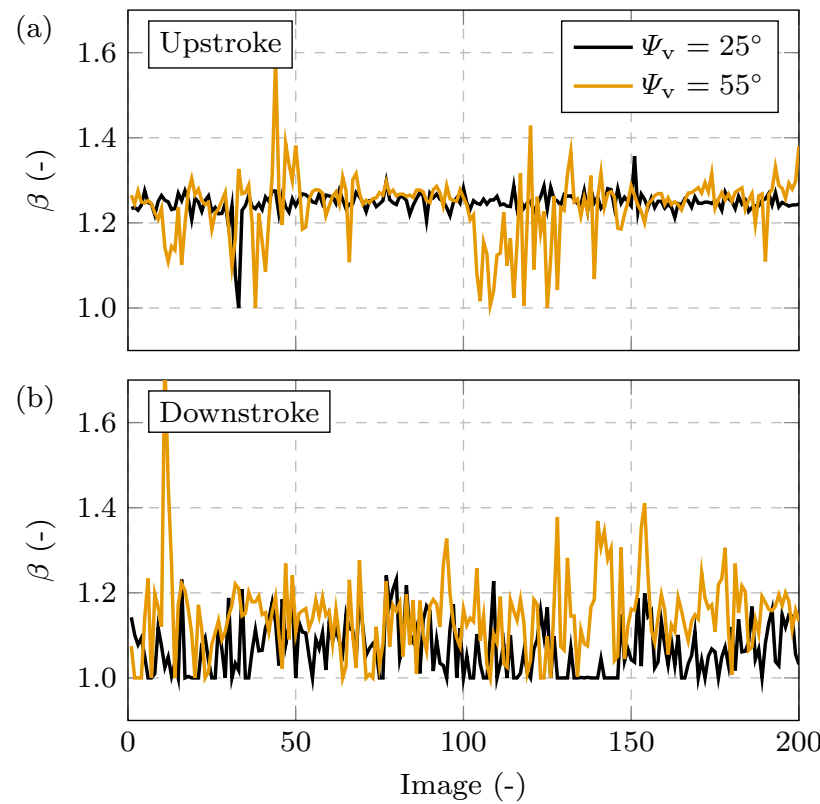

Fig. 21 Turbulence factor $\beta$ of individual fits (Vatistas, $n=1$ ) for each image at vortex ages of $\Psi_{\mathrm{v}}=25^{\circ}$ and $55^{\circ}$. Upstroke $(t / T=0.26)(\mathbf{a})$ and downstroke $(t / T=0.76)(\mathbf{b})$ $\int \frac{V_{\theta}^{2}}{r} \mathrm{~d} r=\frac{\gamma p_{\infty}}{(\gamma-1) \rho_{\infty}^{\gamma}} \rho^{\gamma-1}+$ const.

which links the radial swirl velocity profile of a vortex to its radial density distribution. Bagai and Leishman solved the integral $\int V_{\theta}^{2} / r \mathrm{~d} r$ for the Vatistas 1991 model (Vatistas et al. 1991) for $n=\infty, n=1$, and $n=2$ and provided the equations for the respective density distributions according to Eq. (8).

Since the more recent Vatistas2015 model shows a better agreement with the PIV data of the current study, the respective integrals $\int V_{\theta}^{2} / r \mathrm{~d} r$ have been derived for $n=1$ and $n=2$, following the approach of Bagai and Leishman:

Vatistas2015, $n=1$ :

$\int \frac{V_{\theta}^{2}}{r} \mathrm{~d} r=-\frac{1}{2} V_{\theta, \max }^{2}\left(\frac{(1+\beta)^{1+\beta}}{1+\beta\left(\frac{r}{r_{\mathrm{c}}}\right)^{2}}\right)^{\frac{1}{\beta}}+$ const.

Vatistas2015, $n=2$ :

$$
\begin{array}{r}
\int \frac{V_{\theta}^{2}}{r} \mathrm{~d} r=\frac{1}{2} V_{\theta, \max }^{2} r^{2}\left(r_{\mathrm{c}}^{2}\left(\frac{1+\beta}{r_{\mathrm{c}}^{4}}\right)^{\frac{1+\beta}{2}}\right)^{\frac{1}{\beta}} \\
\cdot{ }_{2} F_{1}\left(\frac{1}{2}, \frac{1+\beta}{2 \beta} ; \frac{3}{2} ;-\frac{\beta r^{4}}{r_{\mathrm{c}}^{4}}\right)+\text { const. }
\end{array}
$$

The solution for $n=2$ uses the hypergeometric function $\left({ }_{2} F_{1}\right)$ since a closed analytical solution is non-existent.

The two integrals can be used in combination with Eq. (8) to obtain the density distributions. The constants of integration can then be found using the boundary condition $\rho(r) \rightarrow \rho_{\infty}$ for $r \rightarrow \infty$, as also stated by Bagai and Leishman (Bagai and Leishman 1993).

The integral $\int V_{\theta}^{2} / r \mathrm{~d} r$ can also be computed numerically based on the PIV swirl velocity. An advantage of using the analytical integral of the best fitting vortex model is evident in the core region. A suitable fit based on the velocity outside of the particle void also provides reliable information inside the vortex core, despite a lack of sufficient particle images in this region.

To link the PIV velocities to the shift measured with BOS, two steps are necessary:

1. Derive the density distribution based on the PIV data and Eq. (8), either by using the analytical integral of the best fitting vortex model or by the numerical integral of the PIV data itself.

2. Calculate the expected BOS shift from the density distribution according to the Gladstone-Dale relation (Liepmann 2013) and with the sensitivity of the utilized BOS setup $S$ (see Eq. (2)). 


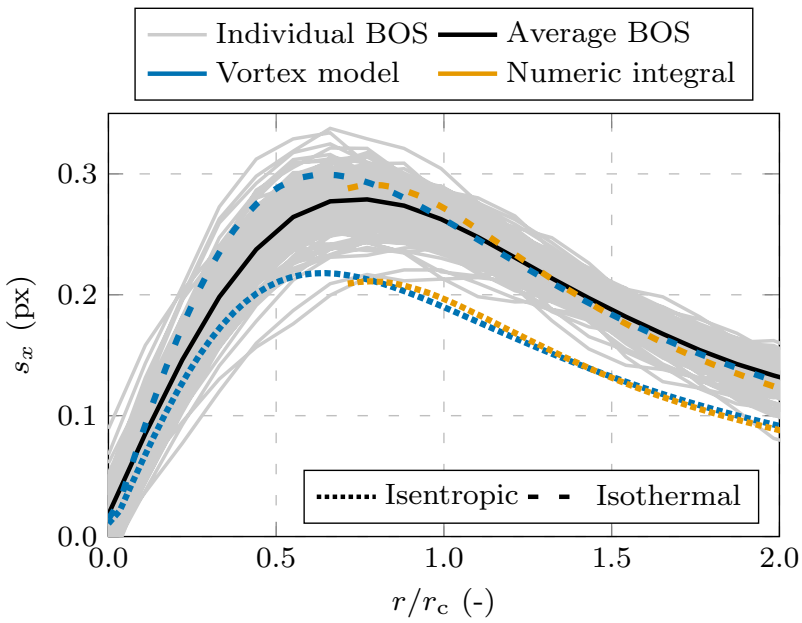

Fig. 22 Individual and average shift obtained by BOS and theoretical shift predicted under the assumption of isentropic or isothermal flow

The shift obtained from one of the two BOS cameras for a test case at a vortex age of $\Psi_{\mathrm{v}}=25^{\circ}$ during upstroke $(t / T=0.42)$ is shown in Fig. 22 over the vortex radius $r$, normalized with the vortex core radius $r_{\mathrm{c}}$. The gray lines represent the 200 instantaneous measurements and the black line indicates the resulting average shift. The theoretical shift, calculated based on the analytical integral of the vortex model Vatistas2015 ( $n=1$, see Eq. (9)), is shown as dotted blue graph. The orange dotted graph represents the theoretical shift calculated with the numerical integral of the PIV data. Both curves follow the assumption of isentropic flow in the vortex core, as proposed by Bagai and Leishman (Bagai and Leishman 1993). The expected agreement between the numerical and the analytical integrals can be seen. Due to the particle void, which has the size of $r_{\text {void }}=0.71 r_{\mathrm{c}}$ in the chosen case, no data for the numerical integration are available below this radius.

The comparison of the predicted shift (dotted lines) and the measured data from BOS reveals an underprediction of the measured data, which is observed for all measured cases in the current study. By using only the experimental data, it is not clear whether the underprediction is caused by the calculation of the density distribution based on the approach by Bagai and Leishman (Bagai and Leishman 1993) (step 1) or by the calculation of the shift utilizing the geometric sensitivity factor $S$ (step 2). To fill this gap, corresponding CFD data are used.

The pressure distribution inside a vortex from CFD is depicted in Fig. 23a over the vortex radius normalized with the vortex core radius $r / r_{\mathrm{c}}$. The expected pressure deficit in the vortex core is present. Figure 23b shows the resulting polytropic exponent $n_{\mathrm{p}}$ derived by Eq. (7), based on the pressure and the density from the simulation (in black). It can be seen that the polytropic exponent in the vortex core is well

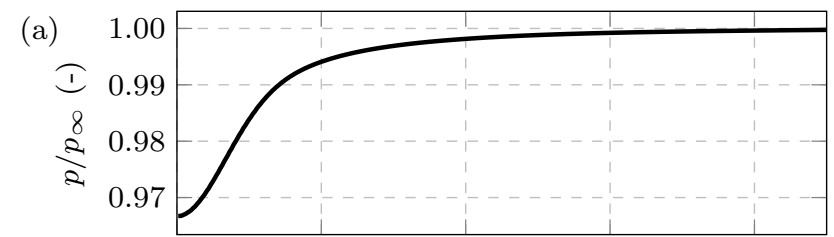

(b)
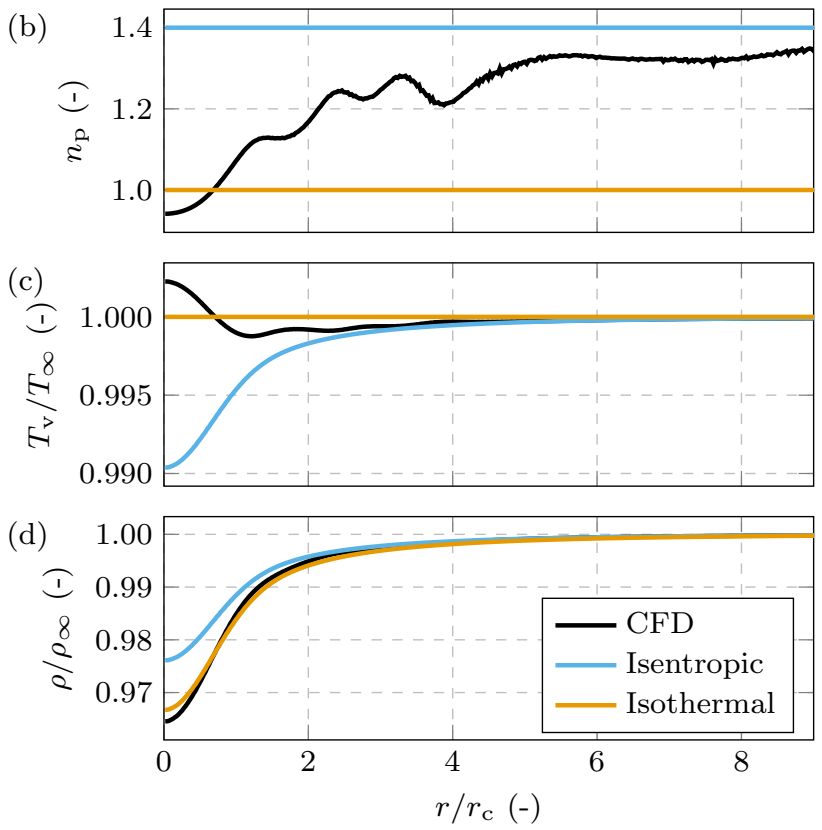

Fig. 23 Pressure $p$, polytropic exponent $n_{\mathrm{p}}$, temperature $T_{\mathrm{v}}$, and density $\rho$ from CFD as well as based on assumptions regarding different polytropic exponents. All plotted against the vortex radius $r$

below the isentropic case $\left(n_{\mathrm{p}}=\gamma=1.4\right)$ and tends toward $n_{\mathrm{p}}=1$ (the isothermal case) and even a bit lower. The temperature distribution from CFD was calculated based on the pressure and the density from CFD, following the ideal gas law. The data given in Fig. 23c indicate an increase in temperature inside the vortex core (black line). The respective density of the simulation is plotted in Fig. 23d, also in black.

Following the assumption of isentropic flow by Bagai and Leishman (1993), the blue curves in Fig. 23 are obtained. An exponent of $n_{\mathrm{p}}=\gamma=1.4$ leads to a reduction of the temperature in the vortex core, based on the pressure distribution from CFD. Furthermore, the density deficit is underpredicted. Assuming isothermal flow $\left(n_{\mathrm{p}}=1\right)$ instead, the temperature is constant by definition, and the density deficit, as predicted by the CFD, is met more closely. The assumption of isothermal flow, thus, represents the CFD simulation better than isentropic flow. For the computation of a vortex core, Yildirim and Hillier (2012) also assumed isothermal conditions in the vortex core and switched to isentropic flow outside the core.

Due to the finite accuracy of the CFD simulations, the small deviations in the polytropic exponent $n_{\mathrm{p}}$, as seen in Fig. 23b, cannot be interpreted. Nonetheless, the tendency 
toward $n_{\mathrm{p}}=1$ inside the vortex and the expected converging toward $n_{\mathrm{p}}=\gamma=1.4$ outside the vortex are observed for all cases.

When applying the assumption of isothermal flow to the relation between velocity and density by Bagai and Leishman (Eq. (8)) and deriving the theoretical BOS shift from the PIV data in the same way as for the data shown as dotted lines in Fig. 22, the shift distribution indicated as dashed lines in the same figure is obtained. It can be seen that the maximum shift is matched more closely compared to the isentropic case, which supports the argument that the assumption of isothermal flow is transferable from the simulation to the measured data.

\subsection{Vortex size from BOS}

The vortex core radius $r_{\mathrm{c}}$ is defined as the radial location of the maximum swirl velocity. The core radius can also be assessed using the shift distributions measured with BOS. This method is independent from a particle void, unlike PIV, and can be performed with only one camera when the position of the vortex is known.

Figure 24 shows the radial locations of the maximum shift $r_{\max \text { shift }}$ for all measured cases over the core radius $r_{\mathrm{c}}$ extracted from the PIV swirl velocity distributions. Figure 24a shows the values for $r_{\max \text { shift }}$ obtained by Camera 1 of the BOS system and Fig. 24b the values obtained by Camera 2.

In a numerical assessment of the shift and velocity distributions associated with both models (Vatistas 1991 and 2015) and both $n$ values, factors between 0.69 and 0.76 were found to link the core radius $r_{\mathrm{c}}$ and the radius of maximum shift $r_{\max \text { shift }}$. In case of the Vatistas 2015 model with $n=1$, the relation between the core radius $r_{\mathrm{c}}$ and the radius of maximum shift $r_{\max \text { shift }}$ is found to be

$r_{\max \text { shift }}=0.70 r_{\mathrm{c}}$.

Therefore, a linear trend in Fig. 24 with a slope of 0.70 is expected and indicated as gray dashed line. It can be seen that for $r_{\mathrm{c}}>5 \mathrm{~mm}$, the values for $r_{\text {max shift }}$ are found to be above the indicated line, but following the expected slope. For smaller $r_{\mathrm{c}}$, the observed radial location of the maximum shift $r_{\text {max shift }}$ remains constant and, thus, deviates from the expected trend. Around this value, the resolution limit of BOS is reached.

In addition to the observed data, the estimated limits of $\mathrm{CoC} / 2$ and the feature size, which led to zero contrast during the evaluation of the 1951 USAF resolution test chart (see Fig. 7), are also shown in both figures. A relation between the observed resolution limits of both cameras and the
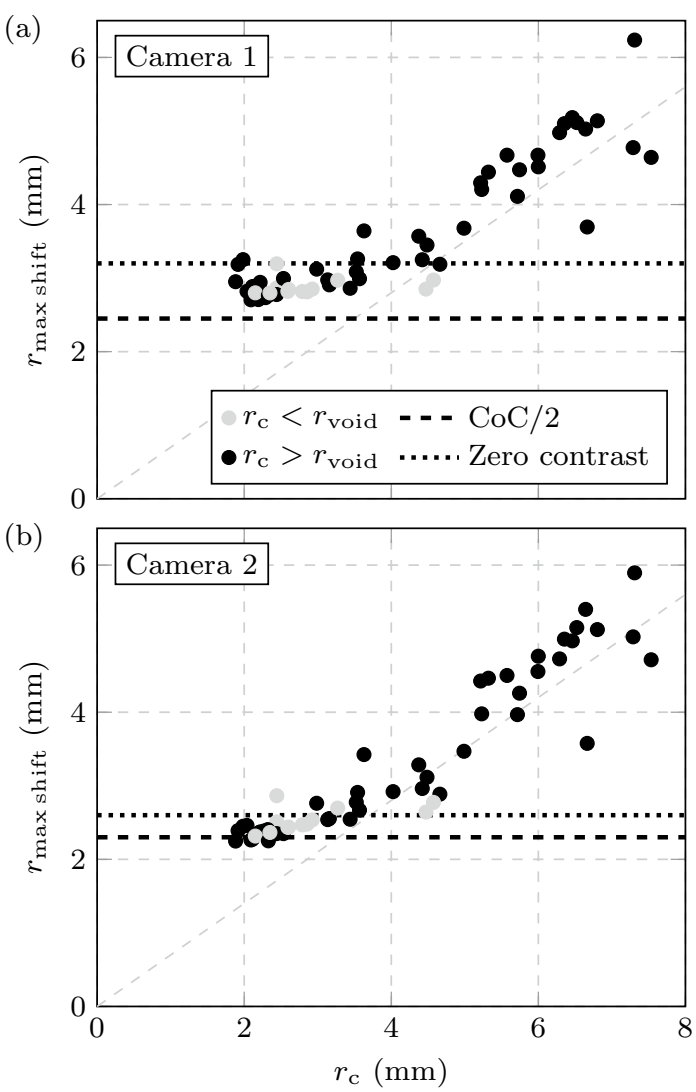

Fig. 24 Radial location of the maximum shift from BOS over the vortex core radius from PIV for all measured cases

deviation of the measured sizes with BOS from the linear trend is evident.

\section{Conclusions}

In this paper, vortices from a sub-scale rotor were investigated by means of BOS and PIV measurements. The measured test cases enable a reconstruction of snapshots of the asymmetrical rotor wake resulting from the cyclic pitch setting. Furthermore, unsteady CFD simulations were used to provide a deeper understanding of the flow physics of the vortices and to link the BOS and PIV results.

The investigation led to the following conclusions:

1. The simultaneous measurements of velocity levels in a plane and a density related shift in a volume both provide data to quantify the mean and the instantaneous vortex positions. The comparison of the two approaches shows good agreement, even for instantaneous measurements.

2. The vortex positions show a scatter around the mean value. The dominant axis and the amplitude of this scat- 
ter were quantified depending on the vortex age as well as on the pitch phase.

3. The density formulation according to Bagai and Leishman (1993) was reassessed for the more recent vortex model by Vatistas from 2015 (Vatistas et al. 2015), which features a factor to account for turbulence inside the vortex and showed better agreement with the current data than the model from 1991 (Vatistas et al. 1991).

4. The link between swirl velocity and density, based on the assumption of isentropic flow by Bagai and Leishman (1993), showed an underprediction of the density compared to the BOS and the CFD data in the current study. The alternative assumption of an isothermal flow (which is based on the findings in the CFD data) results in an excellent agreement between the PIV and the BOS data.

5. Based on the density related shift from the BOS system, the vortex size can also be assessed. For the Vatistas 2015 model with $n=1$, the radius of maximum BOS shift and the vortex core radius can be linked by $r_{\text {max shift }}=0.70 r_{\mathrm{c}}$. The vortex size estimated from the BOS data followed the expected trend above the resolution limit of the BOS setup.

\begin{abstract}
Acknowledgements The experiments of the current investigation were conducted within the framework of the DLR projects "FAST Rescue" and "URBAN Rescue". The authors would like to thank P. Ströer and S. Braun for fruitful discussions. Furthermore, the authors deeply appreciate the reliable support by M. Krebs.
\end{abstract}

Funding Open Access funding enabled and organized by Projekt DEAL.

Open Access This article is licensed under a Creative Commons Attribution 4.0 International License, which permits use, sharing, adaptation, distribution and reproduction in any medium or format, as long as you give appropriate credit to the original author(s) and the source, provide a link to the Creative Commons licence, and indicate if changes were made. The images or other third party material in this article are included in the article's Creative Commons licence, unless indicated otherwise in a credit line to the material. If material is not included in the article's Creative Commons licence and your intended use is not permitted by statutory regulation or exceeds the permitted use, you will need to obtain permission directly from the copyright holder. To view a copy of this licence, visit http://creativecommons.org/licenses/by/4.0/.

\section{References}

Atcheson B, Ihrke I, Heidrich W, Tevs A, Bradley D, Magnor M, Seidel HP (2008) Time-resolved 3D capture of non-stationary gas flows. ACM Trans Graph 27(5), https://doi.org/10.1145/14090 60.1409085

Bagai A, Leishman JG (1993) Flow visualization of compressible vortex structures using density gradient techniques. Exp Fluids 15(6):431-442. https://doi.org/10.1007/bf00191786
Bagai A, Leishman JG (1995) Rotor free-wake modeling using a pseudo-implicit technique - including comparisons with experimental data. J Am Helicopter Soc 40(3):29-41. https://doi.org/ 10.4050/jahs.40.29

Bauknecht A, Ewers B, Wolf C, Leopold F, Yin J, Raffel M (2014) Three-dimensional reconstruction of helicopter blade-tip vortices using a multi-camera BOS system. Exp Fluids 56(1866). https:// doi.org/10.1007/s00348-014-1866-6

Bauknecht A, Ewers B, Schneider O, Raffel M (2015) Aerodynamic results from the star hover test: an examination of the active twist actuation. In: 41st European rotorcraft forum

Bauknecht A, Ewers B, Schneider O, Raffel M (2017) Blade tip vortex measurements on actively twisted rotor blades. Exp Fluids 58(5):49. https://doi.org/10.1007/s00348-017-2312-3

Berger K, Ihrke I, Atcheson B, Heidrich W, Magnor MA (2009) Tomographic 4D reconstruction of gas flows in the presence of occluders. Proc Vis Model Vis Workshop 2009:29-36

Bhagwat MJ, Ramasamy M (2012) Effect of tip vortex aperiodicity on measurement uncertainty. Exp Fluids 53(5):1191-1202. https:// doi.org/10.1007/s00348-012-1348-7

Braukmann JN, Wolf CC, Goerttler A, Raffel M (2020) Blade tip vortex system of a rotor with cyclic pitch. AIAA J 58(7):2869-2880. https://doi.org/10.2514/1.j058678

Dalziel SB, Hughes GO, Sutherland BR (2000) Whole-field density measurements by 'synthetic schlieren.' Exp Fluids 28(4):322-335. https://doi.org/10.1007/s003480050391

De Gregorio F, Visingardi A, Iuso G (2021) An experimental-numerical investigation of the wake structure of a hovering rotor by piv combined with a gamma 2 vortex detection criterion. Energies 14(9):2613. https://doi.org/10.3390/en14092613

Debernardis N (2020) BOS-based three-dimensional reconstruction of rotor blade tip vortices. Master's thesis, Politecnico Di Milano, Dipartimento di Scienze e Tecnologie Aerospaziali

Goerttler A, Braukmann JN, Wolf CC, Gardner AD, Raffel M (2020) Blade tip-vortices of a four-bladed rotor with axial inflow. J Am Helicopter Soc 65(4):1-13. https://doi.org/10.4050/jahs.65. 042002

Gray RB (1956) An aerodynamic analysis of a single bladed rotor in hovering and low speed forward flight as determined from smoke studies of the vorticity distribution in the wake. $\mathrm{PhD}$ thesis, Princeton Univerity, Princeton, NJ, USA, https://doi.org/10. 21236/ad0122600

Greenleaf AR (1950) Photographic optics. Macmillan, NewYork, USA

Heineck J, Kushner L, Schairer E, Walker LA (2010) Retroreflective background oriented schlieren (RBOS) as applied to full-scale UH-60 blade tip vortices. In: American helicopter society aeromechanics specialists conference, pp 722-726

Heineck JT, Yamauchi GK, Wadcock AJ, Lorenco L, Abrego A (2000) Application of three-component PIV to a hovering rotor wake. In: American helicopter society 56th annual forum, American Helicopter Society, pp 1-6

Kaufmann W (1962) Über die Ausbreitung kreiszylindrischer Wirbel in zähen (viskosen) Flüssigkeiten. Ingenieur-Archiv 31(1):1-9. https://doi.org/10.1007/bf00538235

Lasch P, Naumann D (2006) Spatial resolution in infrared microspectroscopic imaging of tissues. Biochimica et Biophysica Acta (BBA) - Biomembranes 1758(7):814-829. https://doi.org/10. 1016/j.bbamem.2006.06.008

Liepmann HW (2013) Elements of gas dynamics. Dover Publications, NewYork, USA

McAlister KW (2004) Rotor wake development during the first revolution. J Am Helicopter Soc 49(4):371-390. https://doi.org/10. 4050/jahs.49.371

Meier GEA (1999) Patentanmeldung Hintergrund-Schlierenmessverfahren. German pat. DE19942856A1 
Meijering E, Jacob M, Sarria J, Steiner P, Hirling H, Unser M (2004) Design and validation of a tool for neurite tracing and analysis in fluorescence images. Cytom Part A J Int Soc Anal Cytol 58(2):167-176. https://doi.org/10.1002/cyto.a.20022

Menter F (1993) Zonal two equation k- $\omega$ turbulence models for aerodynamic flows. In: 23rd fluid dynamics, plasmadynamics, and lasers conference, American Institute of Aeronautics and Astronautics, https://doi.org/10.2514/6.1993-2906

Milluzzo J, Leishman JG (2016) Fluid dynamics of the helicoidal wake sheets trailed from a hovering rotor. $\mathrm{J}$ Am Helicopter Soc 61(1):1-17. https://doi.org/10.4050/JAHS.61.012002

Mula SM, Stephenson J, Tinney CE, Sirohi J (2011) Vortex jitter in hover. In: American helicopter society southwest region technical specialists's meeting

Mulleners K, Raffel M (2011) The onset of dynamic stall revisited. Exp Fluids 52(3):779-793. https://doi.org/10.1007/s00348-011-1118-y

Nicolas F, Todoroff V, Plyer A, Besnerais GL, Donjat D, Micheli F, Champagnat F, Cornic P, Sant YL (2015) A direct approach for instantaneous 3D density field reconstruction from backgroundoriented schlieren (BOS) measurements. Exp Fluids 57(13), https://doi.org/10.1007/s00348-015-2100-x

Overmeyer AD (2015) A rotor tip vortex tracing algorithm for image post-processing. In: American Helicopter Society 71st Annual Forum, Virginia Beach, USA

Raffel M (2015) Background-oriented schlieren (BOS) techniques. Exp Fluids 56(60), https://doi.org/10.1007/s00348-015-1927-5

Raffel M, Richard H, Meier GEA (2000a) On the applicability of background oriented optical tomography for large scale aerodynamic investigations. Exp Fluids 28(5):477-481. https://doi.org/10.1007/ s003480050408

Raffel M, Tung C, Richard H, Yu Y, Meier GEA (2000b) Background oriented stereoscopic schlieren (BOSS) for full-scale helicopter vortex characterization. In: 9th international symposium on flow visualization, Lisbon, Portugal

Raffel M, Bauknecht A, Ramasamy M, Yamauchi GK, Heineck JT, Jenkins LN (2017) Contributions of particle image velocimetry to helicopter aerodynamics. AIAA J 55(9):2859-2874. https://doi. org/10.2514/1.J055571

Richard H, Raffel M, Rein M, Kompenhans J, Meier GEA (2000) Demonstration of the applicability of a background oriented schlieren (BOS) method. In: 10th international symposium on applications of laser techniques to fluid mechanics, Lisbon, Portugal

Schairer E, Kushner L, Heineck J (2013) Measurements of tip vortices from a full-scale UH-60A rotor by retro-reflective background oriented schlieren and stereo photogrammetry. In: American helicopter society 69th annual forum, vol 4, pp 2843-2862

Schwarz C, Bauknecht A, Wolf CC, Coyle A, Raffel M (2020) A fullscale rotor-wake investigation of a free-flying helicopter in ground effect using BOS and PIV. J Am Helicopter Soc 65(3):1-20. https://doi.org/10.4050/jahs.65.032007
Schwermer T, Richter K, Raffel M (2016) Development of a rotor test facility for the investigation of dynamic stall. In: New results in numerical and experimental fluid mechanics $X$, notes on numerical fluid mechanics and multidisciplinary design, vol 132, Springer International Publishing, Cham, Switzerland, pp 633673,https://doi.org/10.1007/978-3-319-27279-58

Scully M, Sullivan JP (1972) Helicopter rotor wake geometry and airloads and development of laser doppler velocimeter for use in helicopter rotor wakes. Technical report, 179, NASA

Settles GS (2006) Schlieren and shadowgraph techniques. Springer Verlag Berlin Heidelberg, Berlin, Germany

Tokgoz S, Geisler R, van Bokhoven LJA, Wieneke B (2012) Temperature and velocity measurements in a fluid layer using background-oriented schlieren and PIV methods. Meas Sci Technol 23(11):115302. https://doi.org/10.1088/0957-0233/23/11/115302

Travin A, Shur M, Strelets M, Spalart PR (2002) Physical and numerical upgrades in the detached-eddy simulation of complex turbulent flows. In: Advances in LES of complex flows, Springer Netherlands, Dordrecht, The Netherlands, pp 239-254, https:// doi.org/10.1007/0-306-48383-1_16

Uluocak S, Perçin M, Uzol O (2021) Experimental investigation of tip anhedral effects on the aerodynamics of a model helicopter rotor in hover. Aero Sci Technol 113:106671. https://doi.org/10.1016/j. ast.2021.106671

van der Wall BG, Richard H (2006) Analysis methodology for 3C-PIV data of rotary wing vortices. Exp Fluids 40(5):798-812. https:// doi.org/10.1007/s00348-006-0117-x

Vatistas GH, Kozel V, Mih WC (1991) A simpler model for concentrated vortices. Exp Fluids 11(1):73-76. https://doi.org/10.1007/ bf00198434

Vatistas GH, Panagiotakakos GD, Manikis FI (2015) Extension of the n-vortex model to approximate the effects of turbulence. J Aircraft 52(5):1721-1725. https://doi.org/10.2514/1.c033238

Wieneke B, Pfeiffer K (2010) Adaptive PIV with variable interrogation window size and shape. In: 15th international symposium on applications of laser techniques to fluid mechanics, Lisbon, Portugal

Wolf CC, Braukmann JN, Stauber W, Schwermer T, Raffel M (2019) The tip vortex system of a four-bladed rotor in dynamic stall conditions. J Am Helicopter Soc 64(2):1-14. https://doi.org/10.4050/ jahs.64.022005

Yildirim E, Hillier R (2012) Numerical modelling of the impulsive orthogonal cutting of a trailing vortex. Shock Waves 23(4):369379. https://doi.org/10.1007/s00193-012-0427-2

Publisher's Note Springer Nature remains neutral with regard to jurisdictional claims in published maps and institutional affiliations. 